\title{
Chiral Symmetries in Nuclear Physics
}

D. Zschiesche ${ }^{1}$, P. Papazoglou ${ }^{1}$, S. Schramm ${ }^{1,2}$, Ch. Beckmamn ${ }^{1}$, J. Schaffner-Bielich ${ }^{3}$, H. Stöcker ${ }^{1}$, and W. Greiner ${ }^{1}$

1 Institut für Theoretische Physik, Postfach 111932 , D-600i-4 Frankfurt am Main, Germany

2 GSI Darmstadt, Postfach 110552 , D-6.1220 Darmstadt. Cimmany

3 Riken BNL Research Center, Brookhawen National Laboratory,

Upton. New York 11973, USA

Abstract. The theoretical concepts of a chirally symmotric moson firzld thererv

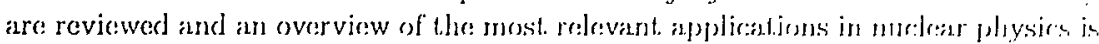

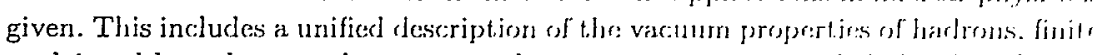
nuclei and hot, dense and strange nuclear matter in an extended chiral SC $(3)_{t}$, $\mathrm{SU}(3)_{R} \sigma-\omega$ model.

\section{General Ideas}

Quantum chromodynamics (QCD) is the accepted theory of strong interactions, but for low energies it is not perturbatively solvable. One idea to overcome this problem is lattice gauge theory, where QCD is solved numerically on a finite space-time lattice. So far, lattice gauge theory is not, able to describe finite nuclei or dense nuclear matter (finite chemical potential) [1]. A different approach has been followed in nuclear physics for several years that is well known from other disciplines, the idea of constructing effective theories. In this concept only the relevant degrees of freedom for the problem are considered to construct a simplified model which is solvable, but contains the interesting and essential characteristics of the full theory. For the cass of strong interactions this means that one considers the hadrons as relevant degrees of freedom instead of quarks, and the bosonic fields are mesons instead of gluons. There exist several models which successfully describe nuclear matter and finite nuclei in such a way (2-4]. Especially the Walecki morlol (QHD) and its extensions (QHD II, non-linear Walecka model) have ber:!1 very successful and widely used for the description of hadronic mallerer anrl finite nuclei. These models are relativistic quantum field theories of baryons and mesons, but they do not consider some of the essential features of QCD. like approximate $\mathrm{SU}(3) \times \mathrm{SU}(3)$ chiral symmetry or broken scale invariance. This lead us to construct an extended chiral $\mathrm{SU}(3) \sigma-\omega$ model of hadrons and mesons, which on the one hand incorporates the successful idea that the: strong interaction is mediated by scalar and vector mesons and which on the other hand contains the relevant symmetries of QCD. In this chapter the basic concepts, the Lagrangian and the main applications of this model will be reported. In Sect. 2 we discuss the motivation for using chiral symmetry in 
effective models for strongly interacting matter. Section 3 shows the representations and transformations of the chiral $\mathrm{SU}(3) \times \mathrm{SU}(3)$ group. The non-linear realization of chiral symmetry and the resulting transformation properties of the relevant degrees of freedom are considered in Sect. 4. The chiral SU(3) Lagrangian is constructed and discussed in Sect. 5 . The equations of motion are solved in the mean-field approximation (Sect. 6). In Sect. 7 we demonstrate that vacuum properties of hadrons and nuclear matter ground-state properties can be described satisfactorily in the chiral SU(3) model. Finally, two main applications are presented. Section 8 shows the results for nuclei, hypernuclei and superheavies and in Sect. 9 the extension to hot and dense hadronic matter is discussed. The conclusions are drawn in Sect. 10.

\section{Why Chiral Symmetry?}

Recently, the general principles of chiral symmetry and broken scale invariance in QCD have received renewed attention at finite baryon densities. There are several reasons for this. First of all in an effective theory of strong interactions, the main features of QCD should be implemented. One important part of these features are symmetries. Lorentz invariance, parity invariance and more are already incorporated in QHD. But chiral symmetry and the broken scale invariance have not been accounted for, even though $\mathrm{SU}(2) \times \mathrm{SU}(2)$ chiral symmetry is a very good symmetry of QCD and SU $(3) \times \mathrm{SU}(3)$ chiral symmetry, even though it is more strongly broken due to the strange-quark mass, can still be considered as an explicitly broken symmetry. In this spirit, models with $\mathrm{SU}(2)_{L} \times \mathrm{SU}(2)_{R}$ symmetry and scale invariance were applied to nuclear matter at zero and finite temperature and to finite nuclei [5-9]. As a new feature, a glueball field $\chi$, the dilaton, was included, which accounted for the broken scale invariance of QCD at tree level through a logarithmic potential [10]. The success of these models established the applicability of this approach to the relativistic description of the nuclear many-body problem. Chiral SU(3) models have been quite successful in describing hadron interactions, e.g. meson-meson interactions can be described very well by using the linear SU(3) $\sigma$ model [11] and kaon-nucleon scattering can be well described using a chiral effective $S U(3)$ Lagrangian $[12,13]$. But these models lack the feature of including the nucleon-nucleon interaction on the same chiral SU(3) basis and therefore do not allow a consistent extrapolation to finite density. Therefore we have extended the chiral effective model to $\mathrm{SU}(3)_{L} \times \mathrm{SU}(3)_{R}$ $[14,15]$. This approach will provide a basis to shed light on the properties of strange hadrons, such as the in-medium properties of the hadrons and the properties of strange hadronic matter, by pinning down the nuclear force in a chirally invariant way. It has been found that hadronic masses of the various $\mathrm{SU}$ (3) multiplets, the nuclear-matter equation of state, finite nuclei, hypernuclei and excited nuclear matter can simultaneously be described reasonably well within a model respecting chiral symmetry.
$3 \quad \mathrm{SU}(3) \times \mathrm{SU}(3)$

The $\sigma$ model has been used extensively in exploring the implications of chiral symmetry in low-energy hadron dynamics. Most of these investigations lave: employed the $S U(2)$ model with mesons and nucleons and the $S U(3) \sigma$ model with mesons only. We require the effective model for hadronic matter to be approximately $\mathrm{SU}(3) \times \mathrm{SU}(3)$-chirally invariant and to include baryons, spin-0) and spin-1 mesons, where the latter are neccssary for non-zero baryon densities. Therefore, in this section we will discuss the transformation properties of spin-0 and spin-1 mesons as well as of the baryons. First, we determine the group representations to the various hadronic multiplets.

\subsection{Representations}

For determining the representations to which the hadrons are assigned, we will look at their quark content. The representations of the hadrons result from the direct product of the quark representations. However, in the Lagrangian, there will be no explicit reference to quarks. For our purpose, they are only used as a pedagogical and mnemonic tool. In the chiral limit, the quarks are massless. Therefore, it is sufficient to consider the two-component spinors

$$
\begin{aligned}
& q_{L}=\frac{1}{2}\left(1-\gamma_{5}\right) q \sim(3,0) \\
& q_{R}=\frac{1}{2}\left(1+\gamma_{5}\right) q \sim(0,3) .
\end{aligned}
$$

Since the quarks are massless, the chirality of the spinors is linked to their spin. On the right-hand side, the quark representations are symbolized by the number of flavors, which is placed left (right) from the somma betweren the brackets for the left (right) subspace.

\section{Mesons}

The mesons visualized as a bound system of a quark and an antirfuark :orrespond to the bilinear form $\bar{q} \mathcal{O}_{q}$ where the $12 \times 12$ matrix $\mathcal{O}$ is the direct. product of the $4 \times 4$ Dirac matrices and the $3 \times 3$ unitary spin matrices $(\mathcal{O}=\Gamma \otimes \lambda)$. For the discussion of the representations we will first suppress the explicit reference to the Gell-Mann matrix $\lambda$.

First, consider the spin-0 mesons. Assuming that they are s-wave bound states, then the only spinless objects we can form are

$\bar{q}_{R} q_{\mathcal{L}}, \quad \bar{q}_{L} q_{R}$.

The combinations $\bar{q}_{L} q_{L}$ and $\bar{q}_{R} q_{R}$ vanish, since the left and right subspaces are orthogonal to each other. The resulting representation is $\left(3.3^{*}\right)$ and 
$\left(3^{*}, 3\right)$, respectively (the antiparticles belong to the conjugate representation). We are thus led to consider nonets of pseudoscalar and scalar particles. For the vector mesons, we have to construct vectorial quantities out of $q_{L}$ and $q_{R}$. Again, if we assume that s-wave bound states are involved, the only vectors which can be formed are

$$
\bar{q}_{L} \gamma_{\mu} q_{L}, \quad \bar{q}_{R} \gamma_{\mu} q_{R} .
$$

This suggests assigning the vector and axial vector mesons to the representation $\left(3 \times 3^{*}, 0\right) \oplus\left(0,3 \times 3^{*}\right)=(8,1) \oplus(1,8)$, coinciding with the tensor properties of the currents conserved in the $\mathrm{SU}(3) \times \mathrm{SU}(3)$ limit $[16,17]$.

\section{Baryons}

The representation of spin- $3 / 2$ baryons can be obtained from the symmetric coupling of three left- or right-handed quarks, $(3 \times 3 \times 3,0)=(10,0)$ or $(0,3 \times$ $3 \times 3)=(0,10)$. For spin-1/2 particles the construction of baryon multiplets from the basic fields $q_{L}$ and $q_{R}$ is not unique. The reason is that a leftor right-handed quark can be added to the spin-0 diquark of one subspace. Consequently, the baryons can be assigned to the representation $\left(3,3^{*}\right)$ and $\left(3^{*}, 3\right)$ or $(8,1)$ and $(1,8)$, respectively. For an explicit construction in terms of quark fields see $[18,19]$.

\subsection{Transformations}

Once the chiral transformation properties of the elementary spinors are known it is straightforward to derive the corresponding transformation properties of the composite fields.

An arbitrary element of $\mathrm{SU}(3) \times \mathrm{SU}(3)$ can be written as

$$
U(\alpha, \beta)=\mathrm{e}^{\left(-\left[\mathrm{i} \alpha_{12} Q^{a}+\mathrm{i} \beta_{a} Q^{5 / a}\right]\right)}=\mathrm{e}^{\left(-\mathrm{i}\left[(\alpha+\beta) \cdot Q_{L}+(\alpha-\beta) \cdot Q_{R}\right]\right)},
$$

where $\alpha$ and $\beta$ are eight-component vectors, and $Q, Q^{5}$ are the vector and axial generators, respectively. The spinor $q_{L}$ transforming under $\mathrm{SU}(3)_{L}$ generated by $Q_{L}$, and $q_{R}$ of $\mathrm{SU}(3)_{R}$ generated by $Q_{R}$, transform infinitesimally as

$$
\begin{aligned}
& q_{i} \longrightarrow q_{i}+\mathrm{i}[(\alpha+\beta) \cdot \lambda / 2]_{i}^{j} q_{j}, \\
& q_{\bar{i}} \longrightarrow q_{\bar{i}}+\mathrm{i}[(\alpha-\beta) \cdot \lambda / 2]_{i}^{j} q_{\bar{j}} .
\end{aligned}
$$

Here, the (un-)barred indices belong to the (right) left subspace. Since the Gell-Mann matrices are Hermitian, the complex-conjugate spinor transforms as

$$
q^{i} \longrightarrow q^{i}-\mathrm{i} q^{j}[(\alpha+\beta) \cdot \lambda / 2]_{j}^{i} .
$$

Knowing the representation of the mesonic and baryonic fields, it is straightforward to derive their transformation properties. They are summarized in
Table 1, where we conveniently express the ficlds in a basis of $3 \times 3$ (icll-Mrmu1 matrices. For example, the spin-0 mesons may be written in the compact form

$$
\begin{aligned}
& \sum_{a=0}^{8}\left(\bar{q}_{L} \lambda^{a} q_{R}+\bar{q}_{L} \lambda^{a} \gamma_{5} q_{R}\right)=\sum_{a=0}^{8}\left(\xi_{a l} \lambda_{l l}+\mathrm{i} \pi_{a l} \lambda_{a l}\right) \quad \because \mid \mathrm{i} / / \quad M \\
& \sum_{a=0}^{8}\left(\bar{q}_{R} \lambda^{a} q_{L}+\bar{q}_{R} \lambda^{a} \gamma_{5} q_{L}\right) \equiv \sum_{a=0}^{8}\left(\xi_{a} \lambda_{1 L}-\mathrm{i} \pi_{a} \lambda_{o}\right)=\Sigma-\mathrm{i} \Pi=M^{i}
\end{aligned}
$$

The first and second rows are connected by the parity transformation. which

\begin{tabular}{|c|c|c|c|}
\hline Hadrons & $J^{P}$ & \multicolumn{2}{|c|}{ Transformations } \\
\hline Spin-0 mesons & $0^{-1}, 0^{-}$ & $L M R^{\dagger}$ & $R M^{\dagger} L^{\dagger}$ \\
\hline Spin-1 mesons & $1^{-}, 1^{+}$ & $L l_{\mu} L^{\dagger}$ & $R r_{\mu} R^{\dagger}$ \\
\hline Baryons (spin- $\frac{1}{2}$-nonet) & $\frac{1}{2}^{+}$ & $L \Psi_{L} R^{\dagger}$ & $R \Psi_{R} L^{\dagger}$ \\
\hline Baryons (spin- $\frac{1}{2}$-octet) & $\frac{1}{2}^{+}$ & $L \Psi_{L} L^{\dagger}$ & $R \Psi_{R} R^{\dagger}$ \\
\hline Baryons (spin- $\frac{3}{2}$-decuplet) & $\frac{3}{2}^{+}$ & $L L L \Delta_{L}$ & $R R R \triangle_{R}$ \\
\hline
\end{tabular}
transforms left-handed quarks to right-handed oncs. In the matrix formula tion this is achieved by taking the adjoint of the expression. Therefore. since scalar and pseudoscalar particles have opposite parity: an imaginary unit i is attarehed tor the psendescalar matrix $I T$

Table 1. Chiral transformations of spin-0 mesons $(M=\Sigma+\mathrm{i} \Pi)$, spin-1 mesons $\left(V_{\mu}=l_{\mu}+r_{\mu}\right.$ and $\left.A_{\mu}=l_{\mu}-r_{\mu}\right)$ and baryons

\section{The Non-linear Realization of Chiral Symmetry}

In some neighborhood of the identity transformation, every group clement $g^{\prime}(x)$ of a compact, semi-simple group $G$ with a subgroup $\mathrm{H}$ can bo docom posed uniquely into a product of the form [20]

$$
g^{\prime}(x)=\exp \left[\mathrm{i} \sum \xi_{a}(x) x_{a}\right] \exp \left[\mathrm{i} \sum \theta_{b}(x) t_{b}\right] \equiv u\left(\xi_{n}(x)\right) h\left(\theta_{b}(x)\right)
$$

where $h\left(\theta_{b}\right)$ is an element of $\mathrm{H}, \xi_{a}$ and $\theta_{b}$ are parameters of the symmetry transformation which are generally space-time-dependent, and $x_{t}$ and $t_{k}$ represent the generators of the group $G$.

For the case of $\mathrm{SU}(3)_{L} \times \mathrm{SU}(3)_{R}$ symmetry, the generators are the vectorial $\left(t_{i}=Q_{i}\right)$ and axial $\left(x_{i}=Q_{i}^{5}\right)$ charges, respectively, and the subgroup is $H=\mathrm{SU}(3)_{V}$. 
For our model, we assume invariance under global SU(3) $L \times \mathrm{SU}(3)_{R}$ transformations

$$
g=\exp \left[\mathrm{i} \sum \alpha_{L}^{a} \lambda_{L a}\right] \exp \left[\mathrm{i} \sum \alpha_{R}^{b} \lambda_{R b}\right] \equiv L\left(\alpha_{L}\right) R\left(\alpha_{R}\right)
$$

Here, the representation of Gell-Mann matrices $\lambda_{L}=\lambda\left(1-\gamma_{5}\right) / 2$ and $\lambda_{R}=$ $\lambda\left(1+\gamma_{5}\right) / 2$ with space-time-independent parameters $\alpha_{L}$ and $\alpha_{R}$ is used.

The product $g u\left(\xi_{a}(x)\right)$ is still an element of $\mathrm{G}$ and can be written as

$$
g \exp \left[\mathrm{i} \sum \xi_{a} x_{a}\right]=\exp \left[\mathrm{i} \sum \xi_{a}^{\prime}\left(g, \xi_{a}\right) x_{a}\right] \exp \left[\mathrm{i} \sum \theta_{b}^{\prime}\left(g, \xi_{a}\right) t_{b}\right]
$$

where, in general, both $\xi_{a}^{\prime}$ and $\theta_{b}^{\prime}$ depend on $g$ and $\xi_{a}$. Let

$$
\tilde{q} \rightarrow D(h) \tilde{q}
$$

be a linear representation of the subgroup $H$ of $G$. Then the transformation

$$
g: \xi \rightarrow \xi^{\prime}, \tilde{q} \rightarrow D\left(\exp \left[\mathrm{i} \sum \theta_{b}^{\prime} t_{b}\right]\right) \tilde{q}
$$

constitutes a non-linear realization of $\mathrm{G}$.

The local parameters of the axial charges are identified with the fields of the pseudoscalar mesons [21]. In the representation of Gell-Mann matrices one has (see also the Appendix)

$$
u\left(\pi_{a}(x)\right)=\exp \left[\frac{\mathrm{i}}{2 \sigma_{0}} \pi^{a}(x) \lambda_{a} \gamma_{5}\right] .
$$

This assignment has the advantage that the pseudoscalar mesons are the parameters of the symmetry transformation. They will therefore only appear if the symmetry is explicitly broken or in terms with derivatives of the fields.

The composition of hadrons in terms of its constituents, the quarks, has to be determined in order to build models with hadronic degrees of freedom. This strategy has been followed, e.g. in [14], and is adopted also here. The transformation properties of the hadrons in the non-linear representation can be derived if the 'old' quarks $q$ are related to the 'new' quarks $\tilde{q}$ of the nonlinear representation.

The quarks of the non-linear representation transform with the vectorial subgroup $\mathrm{SU}(3)_{V}$ in accord with (8). Splitting the quarks into left- and righthanded parts, they can be written as

$$
q_{L}=u \tilde{q}_{L}, \quad q_{R}=u^{\dagger} \tilde{q}_{R}
$$

These equations are connected by parity. The ambiguity in the choice of $h$ is avoided by setting $h=1$. The transformation properties of the pions and the new quarks are found by considering how the old quarks transform

$$
q^{\prime}=L q_{L}+R q_{R}=L u \tilde{q}_{L}+R u^{\dagger} \tilde{q}_{R}
$$

According to $(10)$ (set $g=L$ )

$$
L u=u^{\prime} h \quad ; \quad R u^{\dagger}=u^{\dagger^{\prime}} h,
$$

where the right-hand equation is the parity-transformed left-lmand infuntion Here and in the following, the abbreviations $u \equiv u\left(\pi_{a}(x)\right)$ and $u^{\prime} \equiv u\left(\pi_{a}^{\prime}(x)\right)$ are used. By inserting these relations into (15), one sees that $\tilde{q}$ transforms with $\mathrm{SU}(3)_{V}$ as

$$
\tilde{q}_{L}^{\prime}=h \tilde{q}_{L} \quad ; \quad \tilde{q}_{R}^{\prime}=h \vec{q}_{R} .
$$

According to (10), in general the vector transformation is a local, non-linerat function depending on pseudoscalar mesons, $h=h\left(g, \pi_{a}(x)\right)$. Following $(1(j)$ the pseudoscalar mesons transform non-linearly as

$$
\begin{aligned}
u^{\prime} & =L u h^{\dagger}=h u R^{\dagger}, \\
u^{\dagger} & =L u^{\dagger} L^{\dagger}=R u^{\dagger} h^{\dagger} .
\end{aligned}
$$

The second set of equalities are again due to parity. In contrast to the line realization of chiral symmetry, there is no distinction between the lett and right space. Therefore, only the representations 8 and 1 of the lowest-lying hadrons are possible. The various octets transform accordingly; , g. for the scalar $(X)$, vector $\left(V_{\mu}=l_{\mu}+r_{\mu}\right)$, axial vector $\left(\mathcal{A}_{\mu}=l_{\mu}-r_{\mu}\right)$ and barron $(B, D)$ matrices one has

$$
X^{\prime}=h X h^{\dagger}, V_{\mu}^{\prime}=h V_{\mu} h^{\dagger}, \mathcal{A}_{\mu}^{\prime}=h \mathcal{A}_{\mu} h^{\dagger}, B^{\prime}=h B h^{\dagger}, D^{\prime}=h h h D .
$$

The present, non-linearly transforming, hadronic fields can be obtained from the linearly transforming ones described in [14] by multiplying then with $u(\pi(x))$ and its conjugate (see also [22])

$$
\begin{aligned}
X & =\frac{1}{2}\left(u^{\dagger} M u^{\dagger}+u M^{\dagger} u\right), \quad Y=\frac{1}{2}\left(u^{\dagger} M u^{\dagger}-u M^{\dagger} u\right) . \\
l_{\mu} & =u^{\dagger} \tilde{l}_{\mu} u, \quad r_{\mu}=u \tilde{r}_{\mu} u^{\dagger}, \\
B_{\mathrm{L}} & =u^{\dagger} \Psi_{\mathrm{L}} u, \quad B_{\mathrm{R}}=u \Psi_{\mathrm{R}} u^{\dagger}, \\
D_{\mathrm{L}} & =u u u \Delta_{\mathrm{R}}, \quad D_{\mathrm{R}}=u^{\dagger} u^{\dagger} u^{\dagger} \Delta_{\mathrm{L}} .
\end{aligned}
$$

Here, $M=\Sigma+i \Pi$ and its conjugate contains the nonets of the lincarly transforming scalar $(\Sigma)$ and pseudoscalar $(\Pi)$ mesons, whereas $\tilde{l}_{\mu}, \tilde{r}_{\mu}, \psi_{\mathrm{L}}, \Psi_{\mathrm{R}}$, $\Delta_{\mathrm{I}}$ and $\Delta_{\mathrm{R}}$ are the left- and right-handed parts of the spin-1 mesons, spin-1/2 baryons and spin $3 / 2$ baryons in the linear representation, respoctively.

\section{Lagrangian}

In this section, the various terms of the Lagrangian

$$
\mathcal{L}=\mathcal{L}_{\text {kin }}+\sum_{W=\lambda, Y, V, \mathcal{A}, u} \mathcal{L}_{\mathrm{BW}}+\mathcal{L}_{\mathrm{VP}}+\mathcal{L}_{\text {ver }}+\mathcal{L}_{0}+\mathcal{L}_{\mathrm{SB}}
$$




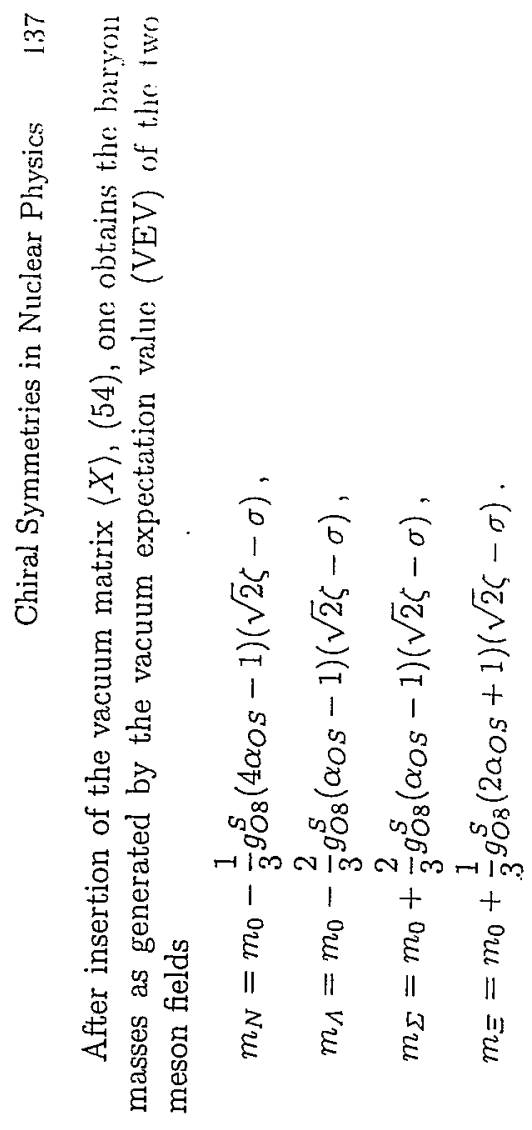

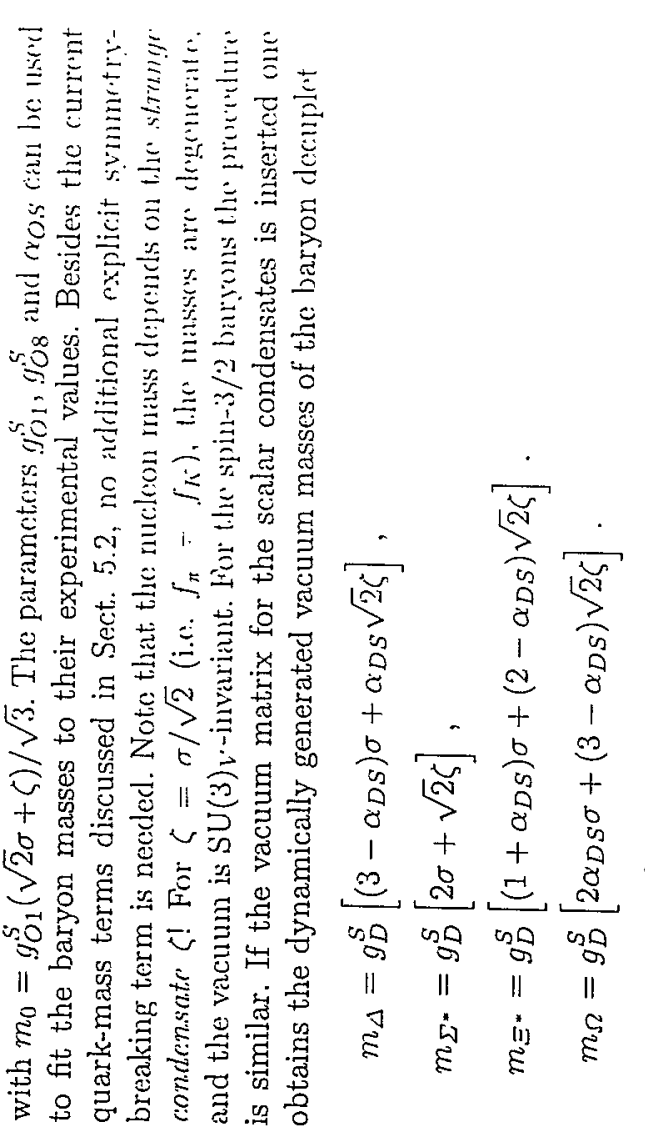

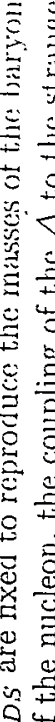

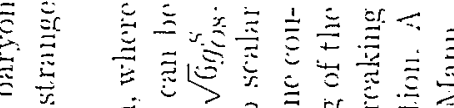

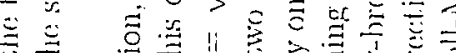

更

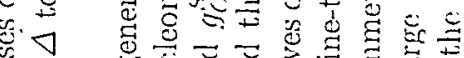

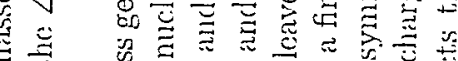

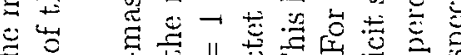

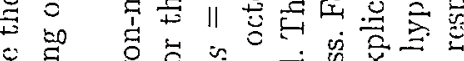

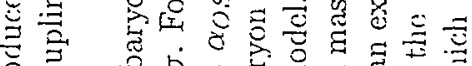

品

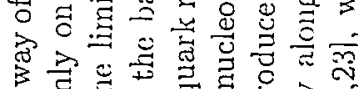

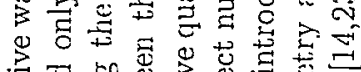

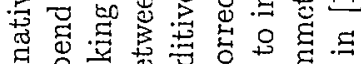

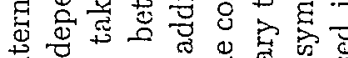

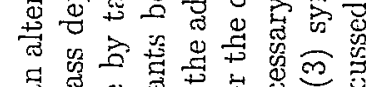

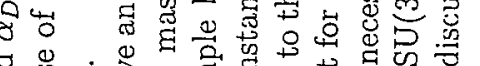

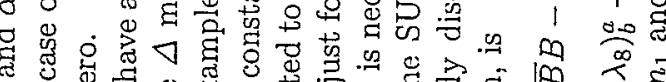

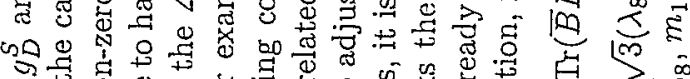

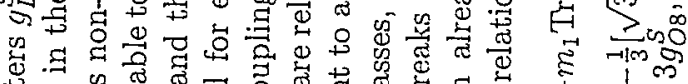

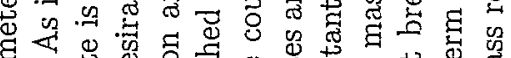

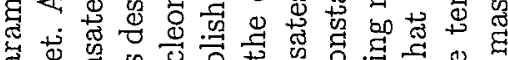

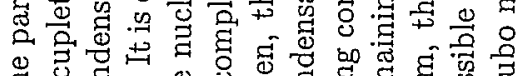

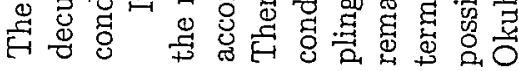

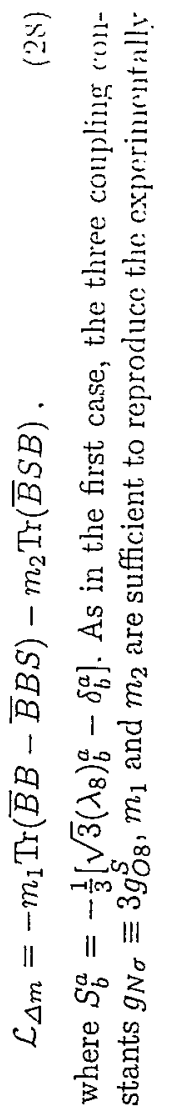

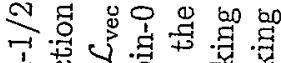

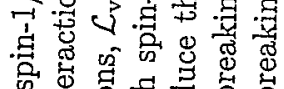

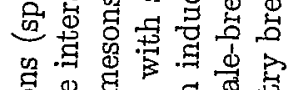
․ㅜㅇㅢ

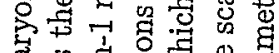

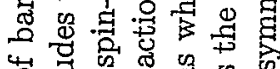

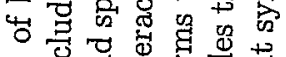
뎍.

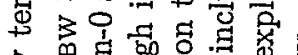

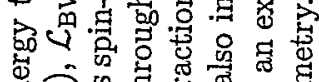
하의

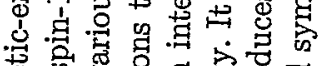

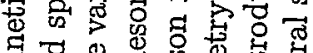

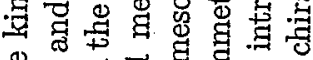

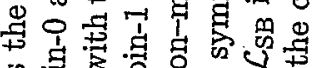

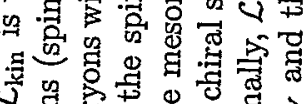

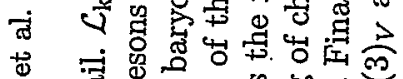

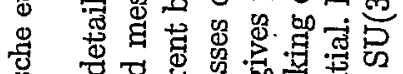

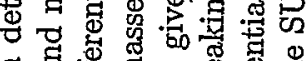

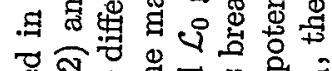

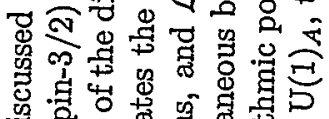

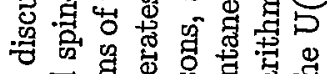

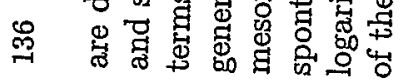

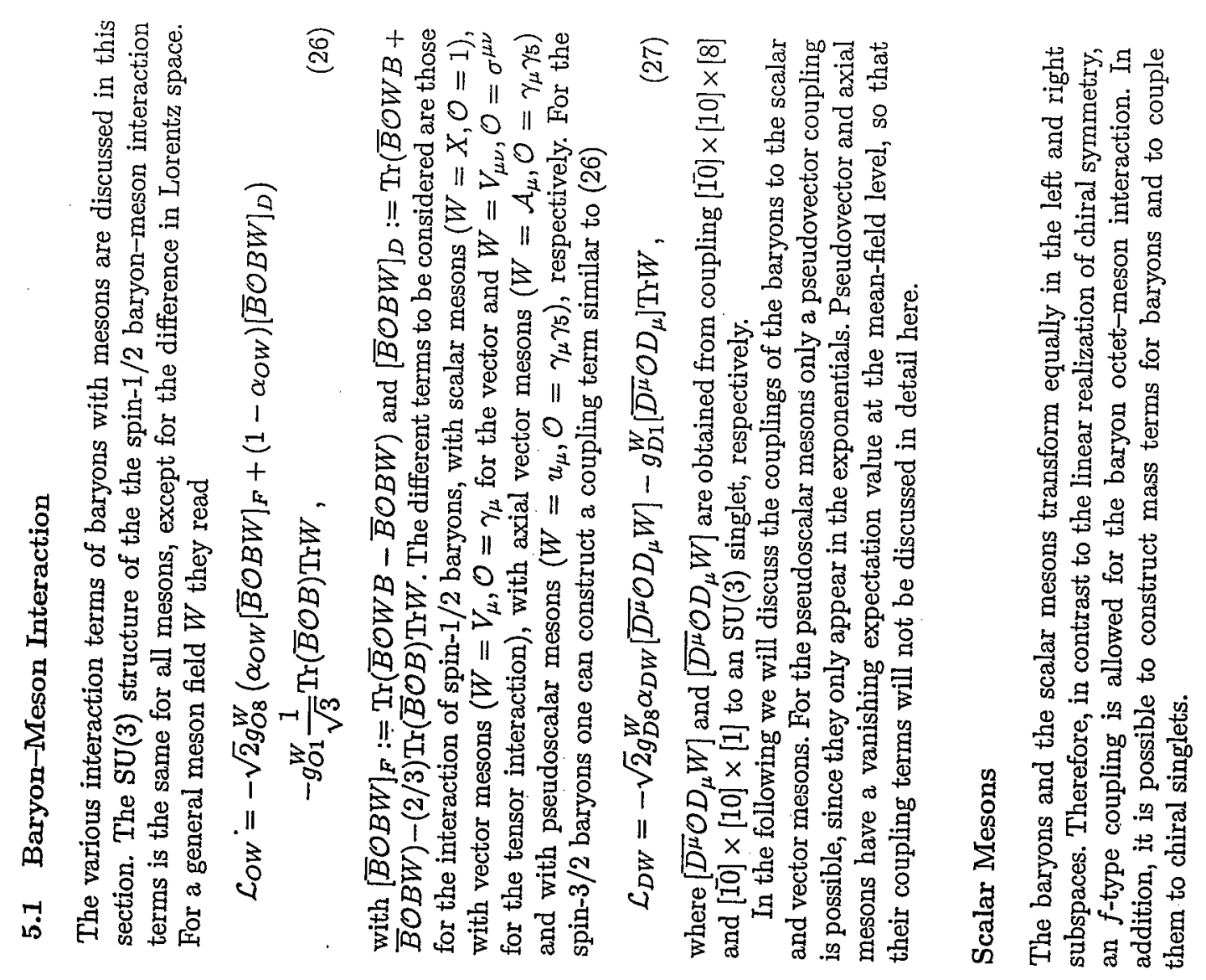


known baryon masses. Explicitly; the baryon masses have the values

$$
\begin{aligned}
& m_{N}=-g_{N \sigma} \sigma, \\
& m_{\Xi}=-\frac{1}{3} g_{N \sigma} \sigma-\frac{2}{3} g_{N \sigma} \sqrt{2} \zeta+m_{1}+m_{2}, \\
& m_{\Lambda}=-\frac{2}{3} g_{N \sigma} \sigma-\frac{1}{3} g_{N \sigma} \sqrt{2} \zeta+\frac{m_{1}+2 m_{2}}{3}, \\
& m_{\Sigma}=-\frac{2}{3} g_{N \sigma} \sigma-\frac{1}{3} g_{N \sigma} \sqrt{2} \zeta+m_{1} .
\end{aligned}
$$

For the baryon decuplet one can choose $\alpha_{D S}=0$ to obtain coupling constants related to the additive quark model. We introduce an explicit symmetrybreaking proportional to the number of strange quarks for a given baryon species. Here we need only one additional parameter $m_{D s}$ to obtain the masses of the baryon decuplet

$$
\begin{aligned}
m_{\Delta} & =g_{\Delta \sigma}[3 \sigma] \\
m_{\Sigma^{*}} & =g_{\Delta \sigma}[2 \sigma+\sqrt{2} \zeta]+m_{D s} \\
m_{\Xi^{*}} & =g_{\Delta \sigma}[1 \sigma+2 \sqrt{2} \zeta]+2 m_{D s} \\
m_{\Omega} & =g_{\Delta \sigma}[0 \sigma+3 \sqrt{2} \zeta]+3 m_{D s}
\end{aligned}
$$

For both versions of the baryon-meson interaction the parameters are fixed to yield the baryon masses of the octet and the decuplet (Tables 2 and 3).

\section{Vector Mesons}

For the spin- $1 / 2$ baryons two independent interaction terms with spin-1 mesons can be constructed in analogy to the interaction of the baryon octet with the scalar mesons. They correspond to the antisymmetric ( $f$-type) and symmetric (d-type) couplings, respectively. From the universality principle [24] and the vector meson dominance model one may conclude that the $d$-type coupling should be small. For most of the fits $\alpha_{V}=1$, i.e. $f$-type coupling, is used. However, a small admixture of $d$-type coupling allows for some fine-tuning of the single-particle energy levels of nucleons in nuclei [15]. As for the case of scalar mesons, for $g_{O 1}^{V}=\sqrt{6} g_{O 8}^{V}$, the strange vector field $\phi_{\mu} \sim \bar{s} \gamma_{\mu} s$ does not couple to the nucleon. The remaining couplings to the strange baryons are then determined by symmetry relations

$$
\begin{aligned}
& g_{N \omega}=\left(4 \alpha_{V}-1\right) g_{O 8}^{V}, \\
& g_{\Lambda \omega}=\frac{2}{3}\left(5 \alpha_{V}-2\right) g_{O 8}^{V}, \quad g_{\Lambda \phi}=-\frac{\sqrt{2}}{3}\left(2 \alpha_{V}+1\right) g_{O 8}^{V}, \\
& g_{\Sigma \omega}=2 \alpha_{V} g_{O 8}^{V}, \quad g_{\Sigma \phi}=-\sqrt{2}\left(2 \alpha_{V}-1\right) g_{O 8}^{V}, \\
& g_{\Xi \omega}=\left(2 \alpha_{V}-1\right) g_{O 8}^{V}, \quad g_{\Xi \phi}=-2 \sqrt{2} \alpha_{V} g_{O 8}^{V} .
\end{aligned}
$$

Table 2. Hadron masses (in $\mathrm{MeV}$ ) for the different fits $C_{1}, C_{2}^{\prime}, C_{3}^{\prime}$

\begin{tabular}{lcccccccc}
\hline \multicolumn{10}{c}{ Spin-0 particle masses } \\
\hline & $m_{\pi}$ & $m_{K}$ & $m_{\eta}$ & $m_{\eta^{\prime}}$ & $m_{a 0}$ & $m_{\kappa^{*}}$ & $m_{\sigma}$ & $m_{f_{11}}$ \\
& $(139)$ & $(495)$ & $(547)$ & $(958)$ & $(980)$ & $(900)$ & & $(980)$ \\
\hline$C_{1}$ & 139.0 & 498.0 & 574.5 & 969.2 & 953.5 & 995.7 & 473.32 & 1039.1 \\
$C_{2}$ & 139.0 & 498.0 & 574.5 & 969.2 & 953.5 & 995.7 & 475.55 & 1039.1 \\
$C_{3}$ & 139.0 & 498.0 & 574.5 & 969.2 & 953.5 & 995.7 & 478.56 & 824.17 \\
& & & & & & & & \\
\hline & & Spin-1 particle masses & Spin-1/2 particle masses \\
\hline & $m_{\omega}$ & $m_{K^{*}}$ & $m_{\rho}$ & $m_{\phi}$ & $m_{N}$ & $m_{A}$ & $m_{\Sigma}$ & $m_{\Xi}$ \\
& $(783)$ & $(892)$ & $(770)$ & $(1020)$ & $(939)$ & $(1115)$ & $(1193)$ & $(1315)$ \\
\hline$C_{1}$ & 783.0 & 863.7 & 770.0 & 1019.0 & 939.0 & 1115.0 & 1196.0 & 1331.5 \\
$C_{2}$ & 783.0 & 863.7 & 770.0 & 1019.0 & 939.0 & 1115.3 & 1196.0 & 1331.5 \\
$C_{3}$ & 783.0 & 863.7 & 770.0 & 1019.0 & 939.0 & 1115.0 & 1196.0 & 1331.5
\end{tabular}

Table 3. Vacuum masses of baryon resonances and baryonic potential depths in nuclear matter

\begin{tabular}{ccccccccc}
\hline & $m_{\Delta}$ & $m_{\Sigma^{*}}$ & $m_{\Xi^{*}}$ & $m_{\Omega}$ & $\frac{m^{*}}{m_{N}}$ & $K(\mathrm{MeV})$ & $U_{N}$ & $l_{i, 1}$ \\
\hline$C_{1}$ & 1232.0 & 1380.0 & 1527.9 & 1675.8 & 0.61 & 276.3 & -71.0 & -28.2 \\
$C_{2}$ & 1232.0 & 1380.2 & 1528.4 & 1676.6 & 0.64 & 266.1 & -68.8 & -30.5 \\
$C_{3}$ & 1232.0 & 1380.0 & 1527.9 & 1675.8 & 0.61 & 285.3 & -71.1 & -28.61 \\
\hline
\end{tabular}

In the limit $\alpha_{V}=1$, the relative values of the coupling constants are rriatiod to the additive quark model via

$$
g_{\Lambda \omega}=g_{\Sigma \omega}=2 g_{\Xi \omega}=\frac{2}{3} g_{N \omega}=2 g_{O 8}^{V}, \quad g_{\Lambda \phi}=g_{\Sigma \phi}=\frac{g_{\Xi} \phi}{2}=\frac{\sqrt{2}}{3} g_{N \omega} .
$$

Note that all coupling constants are fixed once, e.g., $g_{N_{\omega}}$ is specified.

For the coupling of the baryon resonances to the vector mesons wr oltiain the same Clebsch-Gordan coefficients as for the coupling to the scalar mrasons. This leads to the following relations between the coupling constants

$$
\begin{aligned}
g_{\Delta \omega} & =\left(3-\alpha_{D V}\right) g_{D V}, \quad g_{\Delta \phi}=\sqrt{2} \alpha_{D V} g_{D V}, \\
g_{\Sigma^{*} \omega} & =2 g_{D V}, \quad g_{\Sigma^{*} \phi}=\sqrt{2} g_{D V},
\end{aligned}
$$




$$
\begin{aligned}
g_{\Xi^{*} \omega} & =\left(1+\alpha_{D V}\right) g_{D V}, \quad g_{\Xi^{*} \phi}=\sqrt{2}\left(2-\alpha_{D V}\right) g_{D V} \\
g_{\Omega \omega} & =\alpha_{D V} g_{R v}, \quad g_{\Omega \phi}=\sqrt{2}\left(3-\alpha_{D V}\right) g_{D V} .
\end{aligned}
$$

To obtain the coupling of the baryon decuplet to the spin-1 mesons we set $\alpha_{R}=0$, since the strange vector meson $\phi$ should not couple to the $\Delta$ baryon, in analogy to the octet case. The resulting coupling constants again obey the additive quark model constraints

$$
\begin{array}{ll}
g_{\Delta \omega}=\frac{3}{2} g_{\Sigma^{*} \omega}=3 g_{\Xi^{*} \omega}=3 g_{D V}, & g_{\Omega \omega}=0, \\
g_{\Omega \phi}=\frac{3}{2} g_{\Xi^{*} \phi}=3 g_{\Sigma^{*} \phi}=\sqrt{2} g_{\Delta \omega}, & g_{\Delta \phi}=0 .
\end{array}
$$

This means that in the case of the baryon decuplet all coupling constants are again fixed if the overall coupling $g_{D V}$ is specified. Since there is not a vacuum restriction on the $\Delta-\omega$ coupling like in the case of the scalar mesons, we have to consider different constraints. This will be discussed in Sect. 9 .

\subsection{Meson-Meson Interaction}

\section{Vector Meson Masses}

Here we discuss the mass terms of the vector mesons. The simplest scaleinvariant form

$$
\mathcal{L}_{\mathrm{vec}}^{(1)}=\frac{1}{2} m_{V}^{2} \frac{\chi^{2}}{\chi_{0}^{2}} \operatorname{Tr} V_{\mu} V^{\mu}+2 g_{4}^{4} \operatorname{Tr}\left(V_{\mu} V^{\mu}\right)^{2}
$$

implies a mass degeneracy for the meson nonet. The first term of $(30)$ is made scale-invariant by multiplying it with an appropriate power of the glueball field $\chi$ (see Sect. 5.2 for details). To split the masses, one can add the chiral invariant $[25,26]$

$$
\mathcal{L}_{\text {vec }}^{(2)}=\frac{1}{4} \mu \operatorname{Tr}\left[V_{\mu \nu} V^{\mu \nu} X^{2}\right]
$$

A detailed description can be found in [15]. The axial vector meson masses can be described by adding terms analogous to (31). We refrain from discussing them further; see $[25,27]$.

\section{Scalar Mesons}

The non-linear realization of chiral symmetry offers many more possibilities to form chiral invariants: the couplings of scalar mesons with each other are only governed by $\mathrm{SU}(3)_{V}$ symmetry. However, only three kinds of independent invariants exist, namely

$$
I_{1}=\operatorname{Tr} X, \quad I_{2}=\operatorname{Tr} X^{2}, \quad I_{3}=\operatorname{det} X .
$$

All other invariants, $\operatorname{Tr} X^{n}$, with $n \geq 3$, can be expressed as a function of the invariants shown in (32), see [15]. For our calculations, the invariants of (32) are considered as building blocks, from which the different forms of the meson-meson interaction can be constructed. In this chapter we will only discuss the potential of the $\mathrm{SU}(3)$ linear $\sigma$ model [28]. The connection to the models $[5,29]$ and $[2-4]$ is discussed in detail in [15].

\section{Broken Scale Invariance}

The concept of broken scale invariance leading to the trace anomaly in (masiless) $\mathrm{QCD}, \theta_{\mu}^{\mu}=\left(\beta_{\mathrm{QCD}} /(2 g)\right) \mathcal{G}_{\mu \nu}^{c} \mathcal{G}_{a}^{\mu \nu}\left(\mathcal{G}_{\mu \nu}\right.$ is the gluon field-strength tensor of QCD), can be mimicked in an effective Lagrangian at tree level [10] throngh the introduction of the potential

$$
\mathcal{L}_{\text {scale }}=-k_{4} \chi^{4}-\frac{1}{4} \chi^{4} \ln \frac{\chi^{4}}{\chi_{0}^{4}}+\frac{\delta}{3} \chi^{4} \ln \frac{I_{3}}{\operatorname{det}\langle X\rangle} .
$$

The effect ${ }^{1}$ of the logarithmic term $\sim \chi^{4} \ln \chi$ is to break the scale invariance. This leads to the proportionality $\theta_{\mu}^{\mu} \sim \chi^{4}$, as can be seen from

$$
\theta_{\mu}^{\mu}=4 \mathcal{L}-\chi \frac{\partial \mathcal{L}}{\partial \chi}-2 \partial_{\mu} \chi \frac{\partial \mathcal{L}}{\partial\left(\partial_{\mu} \chi\right)}=\chi^{4}
$$

which is a consequence of the definition of the scale transformations :31! This holds only if the meson-meson potential is scalc-invariant, which can l, achieved by multiplying the invariants of scale dimension less than fonr with an appropriate power of the dilaton field $\chi$.

The comparison of the trace anomaly of QCD with that of the effective theory allows for the identification of the $\chi$ field with the gluon condensate

$$
\theta_{\mu}^{\mu}=\left\langle\frac{\beta_{Q C D}}{2 g} \mathcal{G}_{\mu \nu}^{a} \mathcal{G}_{a}^{\mu \nu}\right\rangle \equiv(1-\delta) \chi^{4} .
$$

The parameter $\delta$ originates from the second logarithmic term with the chiral invariant $I_{3}$ (see also [5] for the chiral SU(2) linear $\sigma$ model). An oriontation for the value of $\delta$ may be taken from $\beta_{\text {(a) }}$ at, the one-loop level, with $\Lambda_{\text {, }}$ colors and $N_{\mathrm{f}}$ flavors

$$
\beta_{Q C D}=-\frac{11 N_{\mathrm{c}} g^{3}}{48 \pi^{2}}\left(1-\frac{2 N_{\mathrm{f}}}{11 N_{\mathrm{c}}}\right)+\mathcal{O}\left(g^{5}\right) .
$$

Here the first number in parentheses arises from the (antiscreoning) sellinteraction of the gluons and the second term, proportional to $N_{\mathrm{f}}$, is thr (screening) contribution of quark pairs. Equation (36) suggests the valuc

${ }^{1}$ According to [10], the argument of the logarithm has to be chirally and parity invariant. This is fulfilled by the dilaton, $\chi$, which is both a chiral singlet as woll as a scalar. 
$\delta=2 / 11$ for three flavors and three colors. This value gives the order of magnitude about which the parameter $\delta$ will be varied.

For simplicity, we will also consider the case in which $\chi=\chi_{0}$, where the gluon condensate does not vary with density. We will refer to this case as the frozen glueball limit.

\section{Explicitly Broken Chiral Symmetry}

In order to eliminate the Goldstone modes from a chiral effective theory, explicit symmetry-breaking terms have to be introduced. Here, we use

$$
\mathcal{L}_{\mathrm{SB}}=-\frac{1}{2} m_{\eta_{\mathrm{o}}}^{2} \operatorname{Tr} Y^{2}-\frac{1}{2} \operatorname{Tr} A_{p}\left(u X u+u^{\dagger} X u^{\dagger}\right)-\operatorname{Tr}\left(A_{s}-A_{p}\right) X .
$$

The first term, which breaks the $\mathrm{U}(1)_{A}$ symmetry, gives a mass to the pseudoscalar singlet. The second term is motivated by the explicit symmetrybreaking term of the linear $\sigma$ model

$$
\frac{1}{2} \operatorname{Tr} A_{p}\left(M+M^{\dagger}\right)=\operatorname{Tr} A_{p}\left(u(X+\mathrm{i} Y) u+u^{\dagger}(X-\mathrm{i} Y) u^{\prime}\right),
$$

with $A_{p}=1 / \sqrt{2} \operatorname{diag}\left(m_{\pi}^{2} f_{\pi}, m_{\pi}^{2} f_{\pi}, 2 m_{K}^{2} f_{K}-m_{\pi}^{2} f_{\pi}\right)$ and $m_{\pi}=139 \mathrm{MeV}$, $m_{K}=498 \mathrm{MeV}$. Inserting the spin- 0 meson matrix one obtains

$$
-\mathcal{L}_{\mathrm{SB}}=\frac{\chi^{2}}{\chi_{0}^{2}}\left(m_{\pi}^{2} f_{\pi} \sigma+\left(\sqrt{2} m_{K}^{2} f_{K}-\frac{1}{\sqrt{2}} m_{\pi}^{2} f_{\pi}\right) \zeta\right)
$$

From this the VEV of $\sigma$ and $\zeta$ are fixed by the PCAC relations for the $\pi$ and $K$ mesons

$$
\sigma_{0}=-f_{\pi} \quad \zeta_{0}=\frac{1}{\sqrt{2}}\left(f_{\pi}-2 f_{K}\right) .
$$

For simplicity, $\eta_{0} / \eta_{8}$ mixing is neglected through omitting $Y$ from the second term of (37). If this term is included, we get a mixing angle of $\theta=16^{\circ}$ for parameter set $C_{1}$ [15], which agrees well with experiment, $\theta_{\exp } \approx 20^{\circ}$ from $\eta, \eta^{\prime} \rightarrow \gamma \gamma$.

In the case of SU(3) $V$ symmetry, the quadratic Gell-Mann-Okubo mass formula, $3 m_{\eta_{8}}^{2}+m_{\pi}^{2}-4 m_{K}^{2}=0$, is satisfied.

The third term breaks $\mathrm{SU}(3)_{V}$ symmetry. $A_{s}=\operatorname{diag}(x, x, y)$ can be used to remove the vacuum constraints on the parameters of the meson-meson potential by adjusting $x$ and $y$ in such a way that the terms linear in $\sigma$ and $\zeta$ vanish in the vacuum.

\section{Mean-Field Approximation}

The terms discussed so far involve the full quantum operator fields which cannot be treated exactly. To investigate hadronic matter properties at finite baryon density we adopt the mean-field approximation. This is a nonperturbative relativistic method to solve approximately the nuclear many body problem by replacing the quantum field operators by their classical ( $x$ pectation values (for a recent review see [31]), i.e. the fluctuations around constant vacuum expectation values of the field operators are ueglectered

$$
\begin{aligned}
\sigma(x) & =\langle\sigma\rangle+\delta \sigma \rightarrow\langle\sigma\rangle \equiv \sigma, \quad \zeta(x)=\langle\zeta\rangle+\delta \zeta \rightarrow\langle\zeta\rangle \equiv \zeta, \\
\omega_{\mu}(x) & =\langle\omega\rangle \delta_{0 \mu}+\delta \omega_{\mu} \rightarrow\left\langle\omega_{0}\right\rangle \equiv \omega, \\
\phi_{\mu}(x) & =\langle\phi\rangle \delta_{0 \mu}+\delta \phi_{\mu} \rightarrow\left\langle\phi_{0}\right\rangle \equiv \phi .
\end{aligned}
$$

The fermions are treated as quanlum-mechanical one-particle operators. Tho derivative terms can be neglected and only the time-like component of the vector mesons $\omega \equiv\left\langle\omega_{0}\right\rangle$ and $\phi \equiv\left\langle\phi_{0}\right\rangle$ survive if we assume homogeneous and isotropic infinite baryonic matter. Additionally, due to parity conservation we have $\left\langle\pi_{i}\right\rangle=0$. After performing these approximations, the Lagrangian (25) becomes

$$
\begin{aligned}
\mathcal{L}_{B M}+\mathcal{L}_{B V}= & -\sum_{i} \overline{\psi_{i}}\left[g_{i \omega} \gamma_{0} \omega^{0}+g_{i \phi} \gamma_{0} \phi^{0}+m_{i}^{*}\right] \psi_{i}, \\
\mathcal{L}_{\mathrm{vec}}= & \frac{1}{2} m_{\omega}^{2} \frac{\chi^{2}}{\chi_{0}^{2}} \omega^{2}+\frac{1}{2} m_{\phi}^{2} \frac{\chi^{2}}{\chi_{0}^{2}} \phi^{2}+g_{4}^{4}\left(\omega^{4}+2 \phi^{4}\right), \\
\mathcal{V}_{0}= & \frac{1}{2} k_{0} \chi^{2}\left(\sigma^{2}+\zeta^{2}\right)-k_{1}\left(\sigma^{2}+\zeta^{2}\right)^{2}-k_{2}\left(\frac{\sigma^{4}}{2}+\zeta^{4}\right) \\
& -k_{3} \chi \sigma^{2} \zeta+k_{4} \chi^{4}+\frac{1}{4} \chi^{4} \ln \frac{\chi^{4}}{\chi_{0}^{4}}-\frac{\delta}{3} \ln \frac{\sigma^{2} \zeta}{\sigma_{0}^{2} \zeta_{0}}, \\
\mathcal{V}_{\mathrm{SB}}= & \left(\frac{\chi}{\chi_{0}}\right)^{2}\left[m_{\pi}^{2} f_{\pi} \sigma+\left(\sqrt{2} m_{K}^{2} f_{K}-\frac{1}{\sqrt{2}} m_{\pi}^{2} f_{\pi}\right) \zeta\right],
\end{aligned}
$$

with $m_{i}$ the effective mass of the baryon $i$, which is defined according to Sect. 5.1, and $i=N, \Lambda, \Sigma, \Xi, \Delta, \Sigma^{*}, \Xi^{*}, \Omega$.

Now it is straightforward to write down the expression for the thermodynamical potential of the grand canonical ensemble $\Omega$ per volume $V$ at a given chemical potential $\mu$ and zero temperature

$$
\frac{\Omega}{V}=-\mathcal{L}_{\mathrm{vec}}-\mathcal{L}_{0}-\mathcal{L}_{\mathrm{SB}}-\mathcal{V}_{\mathrm{vac}}-\sum_{i} \frac{\gamma_{i}}{(2 \pi)^{3}} \int \mathrm{d}^{3} k\left[E_{i}^{*}(k)-\mu_{i}^{*}\right]
$$

The vacuum energy $\mathcal{V}_{\text {vac }}$ (the potential at $\rho=0$ ) has been subtracted in order to get a vanishing vacuum energy. $\gamma_{i}$ denote the fermionic spin-isospin degeneracy factors. The single-particle energies are $E_{i}^{*}(k)=\left(k_{i}^{2}+m_{i}^{* 2}\right)^{1 / 2}$ and the effective chemical potentials read $\mu_{i}^{*}=\mu_{i}-g_{w i} \omega-g_{\phi i} \phi$.

The mesonic fields are determined by extremizing $\Omega / V(\mu, T=0)$

$$
\begin{aligned}
\frac{\partial(\Omega / V)}{\partial \chi}= & -\omega^{2} m_{\omega}^{2} \frac{\chi}{\chi_{0}^{2}}+k_{0} \chi\left(\sigma^{2}+\zeta^{2}\right)-k_{3} \sigma^{2} \zeta \\
& +\left(4 k_{4}+1+4 \ln \frac{\chi}{\chi_{0}}-4 \frac{\delta}{3} \ln \frac{\sigma^{2} \zeta}{\sigma_{0}^{2} \zeta_{1}}\right) \chi^{3}
\end{aligned}
$$




$$
\begin{aligned}
& +2 \frac{\chi}{\chi_{0}^{2}}\left[m_{\pi}^{2} f_{\pi} \sigma+\left(\sqrt{2} m_{K}^{2} f_{K}-\frac{1}{\sqrt{2}} m_{\pi}^{2} f_{\pi}\right) \zeta\right]=0, \\
\frac{\partial(\Omega / V)}{\partial \sigma}= & k_{0} \chi^{2} \sigma-4 k_{1}\left(\sigma^{2}+\zeta^{2}\right) \sigma-2 k_{2} \sigma^{3}-2 k_{3} \chi \sigma \zeta-2 \frac{\delta \chi^{4}}{3 \sigma} \\
& +\left(\frac{\chi}{\chi_{0}}\right)^{2} m_{\pi}^{2} f_{\pi}+\sum_{i} \frac{\partial m_{i}^{*}}{\partial \sigma} \rho_{i}^{s}=0, \\
\frac{\partial(\Omega / V)}{\partial \zeta}= & k_{0} \chi^{2} \zeta-4 k_{1}\left(\sigma^{2}+\zeta^{2}\right) \zeta-4 k_{2} \zeta^{3}-k_{3} \chi \sigma^{2}-\frac{\delta \chi^{4}}{3 \zeta} \\
& +\left(\frac{\chi}{\chi_{0}}\right)^{2}\left[\sqrt{2} m_{K}^{2} f_{K}-\frac{1}{\sqrt{2}} m_{\pi}^{2} f_{\pi}\right]+\sum_{i} \frac{\partial m_{i}^{*}}{\partial \zeta} \rho_{i}^{s}=0, \\
\frac{\partial(\Omega / V)}{\partial \omega}= & -\left(\frac{\chi}{\chi_{0}}\right) m_{\omega}^{2} \omega-4 g_{4}^{4} \omega^{3}+\sum_{i} \frac{g_{i \omega}}{\rho_{i}}=0, \\
\frac{\partial(\Omega / V)}{\partial \phi}= & -\left(\frac{\chi}{\chi_{0}}\right) m_{\phi}^{2} \phi-8 g_{4}^{4} \phi^{3}+\sum_{i} \frac{g_{i \phi}}{\rho_{i}}=0 .
\end{aligned}
$$

The scalar densities $\rho_{i}^{s}$ and the vector densities $\rho_{i}$ can be calculated analytically for the case $T=0$, yielding

$$
\begin{aligned}
& \rho_{i}^{s}=\gamma_{i} \int \frac{\mathrm{d}^{3} k}{(2 \pi)^{3}} \frac{m_{i}^{*}}{E_{i}^{*}}=\frac{\gamma_{i} m_{i}^{*}}{4 \pi^{2}}\left[k_{F i} E_{F i}^{*}-m_{i}^{* 2} \ln \left(\frac{k_{F i}+E_{F i}^{*}}{m_{i}^{*}}\right)\right], \\
& \rho_{i}=\gamma_{i} \int_{0}^{k_{F i}} \frac{\mathrm{d}^{3} k}{(2 \pi)^{3}}=\frac{\gamma_{i} k_{F i}^{3}}{6 \pi^{2}} .
\end{aligned}
$$

The energy density and the pressure follow from the Gibbs-Duhem relation, $\epsilon=\Omega / V+\mu_{i} \rho^{i}$ and $p=-\Omega / V$. Applying the Hugenholtz-van Hove theorem [32], the Fermi surfaces are given by $E^{*}\left(k_{F i}\right)=\left(k_{F i}^{2}+m_{i}^{* 2}\right)^{1 / 2}=\mu_{i}^{*}$.

\section{Nuclear Matter}

Here we discuss how the parameters of the effective model are fixed to vacuum and nuclear-matter ground-state properties. Furthermore predicted observables will be discussed.

\subsection{Fixing of Parameters}

The elements of the matrix $A_{p}$ are fixed to fulfill the PCAC relations of the pion and the kaon, respectively. Therefore, the parameters of the chirally invariant potential, $k_{0}$ and $k_{2}$, are used to ensure an extremum in the vacuum. As for the remaining constants, $k_{3}$ is constrained by the $\eta^{\prime}$ mass, and $k_{1}$ is varied to give a $\sigma$ mass of the order of $m_{\sigma}=500 \mathrm{MeV}$. The VEV of the gluon condensate, $\chi_{0}$, is fixed to fit the binding energy of nuclear matter $\epsilon_{0} / \rho-m_{N}=-16 \mathrm{MeV}$ at the saturation density $p_{0}=0.15 \mathrm{fm}^{-3}$. The VEVs of the fields $\sigma_{0}$ and $\zeta_{0}$ are constrained by the decay constants of the pion and the kaon, respectively, see (40). As stated before, the coupling constiant.s of the baryons to the scalar mesons are fitted to the experimental values of the ir vacuum masses. The coupling constant of the spin-1 mesons to the spin-1/2 baryons is chosen to ensure that the nuclear-matter ground-state pressure vanishes. The coupling of the baryon resonances to the spin-1 mesons will be discussed in Sect. 9.

\subsection{Vacuum and Nuclear-Matter Properties}

We will mainly concentrate on the results obtained using three different parameter sets:

- $C_{1}$ : frozen glueball, baryon masses without additional symmetry-breaking:

- $C_{2}$ : frozen glueball, baryon masses including additional symmetry-breaking:

- $C_{3}$ : non-frozen glueball, baryon masses like $C_{1}$.

The values of the parameters can be seen in Table 4.

Table 4. Parameters of the fits (see text)

\begin{tabular}{cccccccc}
\hline & $k_{0}$ & $k_{1}$ & $k_{2}$ & $k_{3}$ & $k_{3 m}$ & $k_{i 1}$ & $33 \delta$ \\
\hline$C_{1}$ & 2.37 & 1.40 & -5.55 & -2.65 & 0 & -0.23 & 2 \\
$C_{2}$ & 2.36 & 1.40 & -5.55 & -2.64 & 0 & -0.23 & 2 \\
$C_{3}$ & 2.35 & 1.40 & -5.55 & -2.60 & 0 & -0.23 & 2 \\
\hline
\end{tabular}

The hadronic masses in the vacuum have reasonable valurs for all fit.s (Tables 2 and 3 )

According to Table 3 , the values of the effective nucleon mass and the compressibility in the medium (at $\rho_{0}$ ) are reasonable. To obtain these values the inclusion of a quartic term for vector mesons (see (30)) was nocessiry: Table 3 shows the nucleon and $\Lambda$ potential in saturated nuclear matter, which are in good agreement with extrapolations from binding energies in nuclei. Using different forms for the mesonic potential one can obtain other successful models for the description of nuclear matter and finite nuclei. This has berm done for the Minnesota model [5] and the Walecka model [15].

\section{Nuclei and Hypernuclei}

As was pointed out in [33], reproducing the nuclear-matter equilibrium point. is not sufficient to ensure a quantitative description of nuclear phenomenology. For this, one has to study the systematics of finite nuclei. 'To apply the 
model to the description of finite nuclei, we also adopt the mean-field approximation. Compared to nuclear matter one has to take additional terms into account for the description of finite nuclei.

- Since one considers now a finite system, the spatial derivatives of the fields have to be taken into account.

- Since the system is not isospin-symmetric any more, the expectation value for the $\rho$ meson does not vanish any more.

- Electromagnetic interactions have to be taken into account.

This leads to the following additional terms for the Lagrangian

$$
\begin{aligned}
& \mathcal{L}_{\text {kin }}^{\prime}=-\mathrm{i} \bar{N} \gamma_{\mu} \partial^{\mu} N-\frac{1}{2} \sum_{\varphi=\sigma, \zeta, \chi, \omega, \rho, A} \nabla_{i} \varphi \nabla^{i} \varphi, \\
& \mathcal{L}_{B V}^{\prime}=-\bar{N} \gamma_{0}\left[g_{N \rho} \tau_{3} \rho_{0}+\frac{1}{2} e\left(1+\tau_{3}\right) A_{0}\right] N, \\
& \mathcal{L}_{\text {vec }}^{\prime}=\frac{1}{2} \frac{\chi^{2}}{\chi_{0}^{2}} m_{\rho}^{2} \rho^{2}+g_{4}^{4}\left(6 \omega^{2} \rho^{2}+\rho^{4}\right) .
\end{aligned}
$$

The resulting Dirac equation for the nucleon and the equation for the photon field are of the form given, e.g., by Reinhard [34]. The densities $\rho_{s}=\langle\bar{N} N\rangle$, $\rho_{B}=\left\langle\bar{N} \gamma_{0} N\right\rangle$ and $\rho_{3}=\left\langle\bar{N} \gamma_{0} \tau_{3} N\right\rangle$ can be expressed in terms of the components of the nucleon Dirac spinors in the usual way [31]. The set of coupled equations is solved numerically in an accelerated-gradient iteration following [35]. Without changing the parameters of the model, the properties of nuclei can readily be predicted.

The charge densities of ${ }^{16} \mathrm{O},{ }^{40} \mathrm{Ca}$ and ${ }^{208} \mathrm{~Pb}$ are quite close to experiment. They exhibit relatively small radial oscillations (Figs. 1,2 and 3), though such oscillations are not seen in the experimental data. ${ }^{2}$ The experimental charge densities are obtained from [37], where a three-parameter Fermi model was used. ${ }^{3}$ The charge radii are close to the experimental values. Figure 4 shows the charge form factor of ${ }^{208} \mathrm{~Pb}$ for parameter set $C_{1}$ in momentum space. For small momenta the agreement with experiment [37] is very good, only for larger momenta are deviations observable but at the same scale as in the non-linear Walecka model [29]. The binding energies of ${ }^{16} \mathrm{O},{ }^{40} \mathrm{Ca}$ and ${ }^{208} \mathrm{~Pb}$ are in reasonable agreement with the experimental data (see Table 5). Nevertheless they are off by approximately $0.5 \mathrm{MeV}$. To correct this, a direct fit to nuclear properties has to be done [38].

\footnotetext{
${ }^{2}$ Similar problems exist also for non-chiral models; for a discussion see $[33,36]$.

${ }^{3} \mathrm{~A}$ more sophisticated model-independent analysis by means of an expansion of the charge distribution as a sum of Gaussians would lead to an even closer correspondence between our results and the experimental data.
}

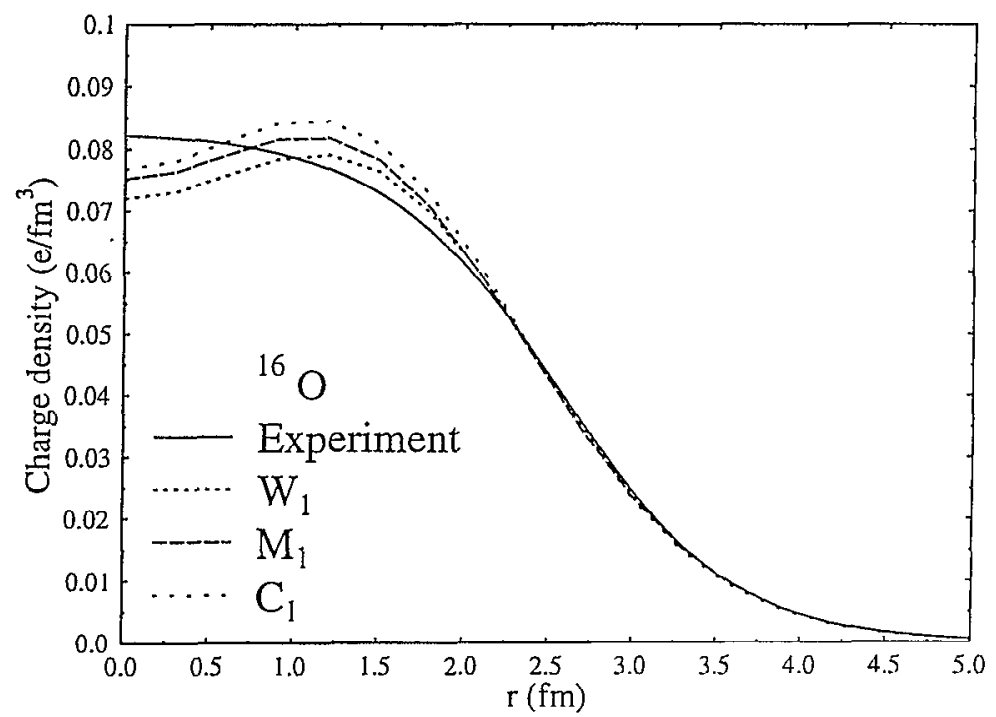

Fig. 1. Charge density for ${ }^{16} \mathrm{O}$ for the parameter sets indicated in [15]. $\left(C_{1}\right)$ chiral SU(3) model. $\left(M_{1}\right)$ chiral SU(2) Minnesota model. $\left(W_{1}\right)$ extended Walecka modcl. The experimental charge density is parameterized with a three-parameter Formi model [37]

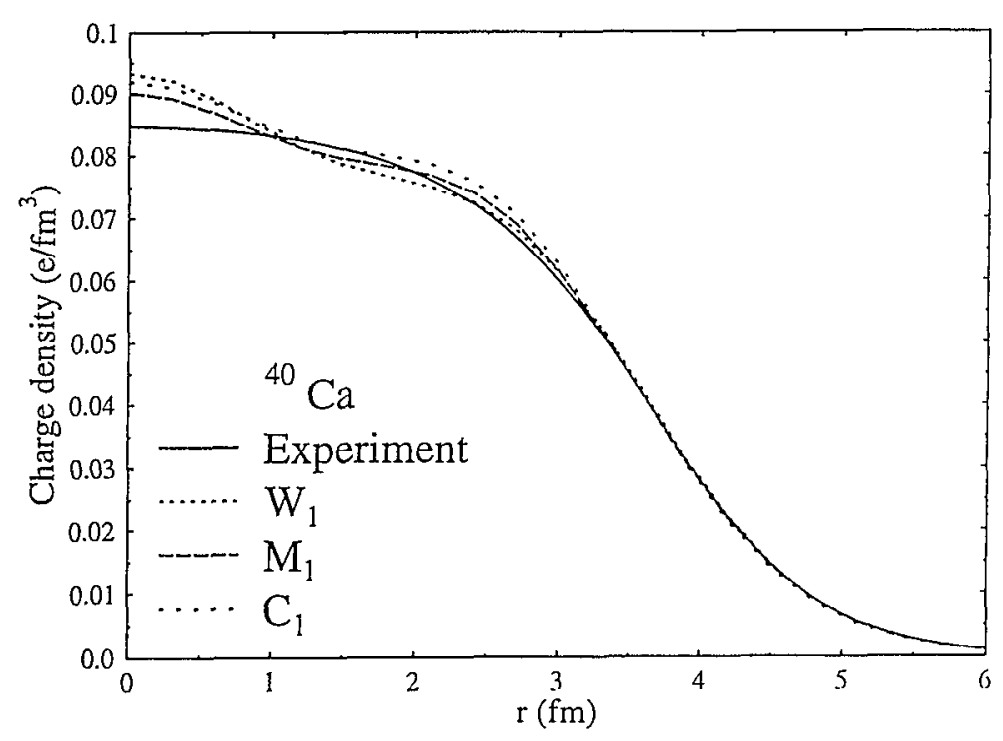

Fig. 2. As for Fig. 1, but for ${ }^{40} \mathrm{Ca}$ 


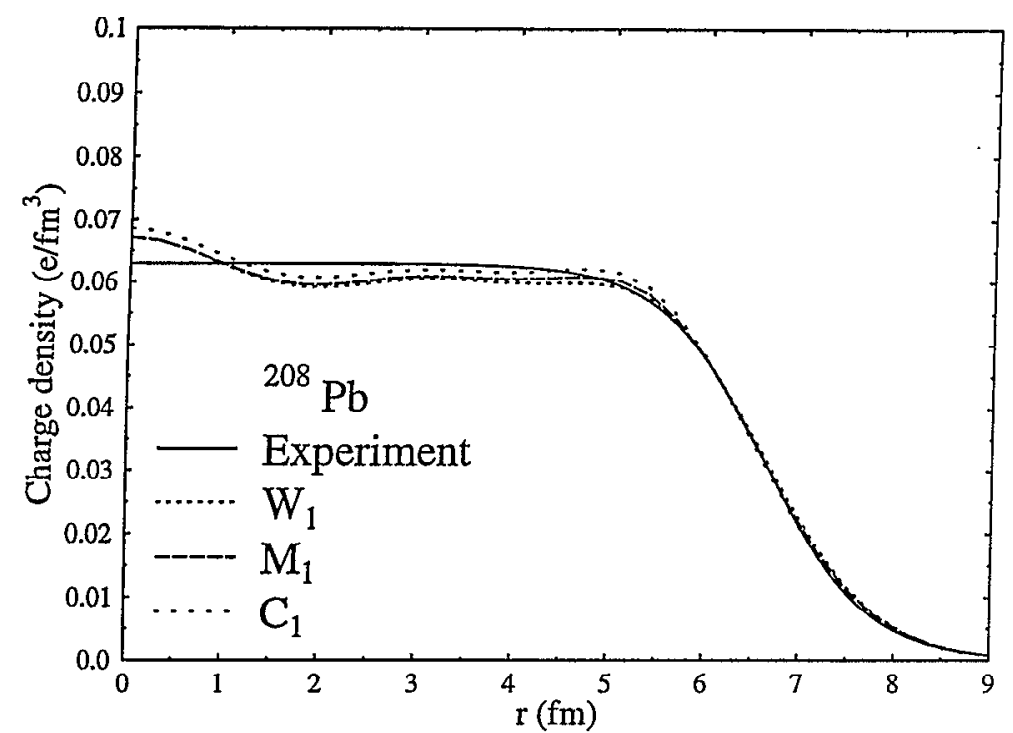

Fig. 3. As for Fig. 1, but for ${ }^{208} \mathrm{~Pb}$

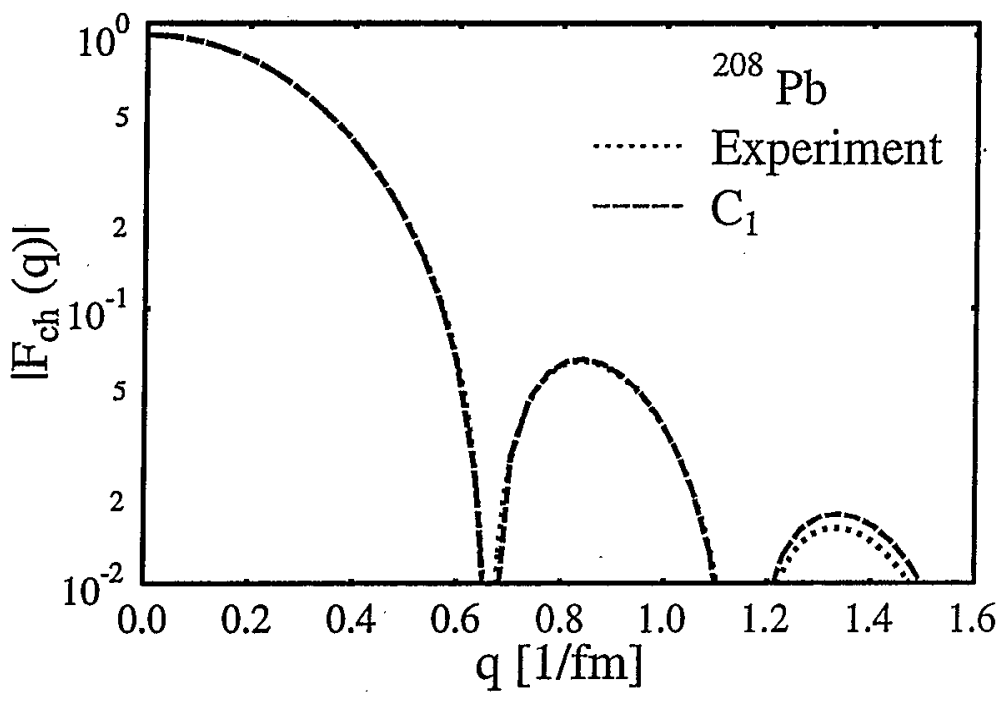

Fig. 4. Charge form factor for ${ }^{208} \mathrm{~Pb}$
Table 5. Bulk properties of nuckei: Prediction and experimential vilumes for himdung: energy $E / A$, charge radius $r_{c / 2}$, and spin-orbit splitting of oxygen $\left({ }^{\text {f. }}()\right.$ with ho $\left.p_{3 / 2}-p_{1 / 2}\right)$, calcium $\left({ }^{40} \mathrm{Ca}\right.$ with $\left.\delta d \equiv d_{5 / 2}-d_{3 / 2}\right)$ and lead $\left({ }^{2(1 \times} \mathrm{p}\right.$ ) w with $\dot{j} d=$ $\left.2 d_{5 / 2}-2 d_{3 / 2}\right)$

\begin{tabular}{|c|c|c|c|c|c|c|c|c|c|}
\hline & \multicolumn{3}{|c|}{${ }^{16} \mathrm{O}$} & \multicolumn{3}{|c|}{${ }^{40} \mathrm{Ca}$} & \multicolumn{3}{|c|}{${ }^{208} \mathrm{~Pb}$} \\
\hline & $E / A$ & $r_{c h}$ & $\delta p$ & $E / A$ & $r_{c h}$ & $\delta d$ & $E / A$ & $r_{c h}$ & $\delta d$ \\
\hline Exp. & -7.98 & 2.73 & $5.5-6.6$ & -8.55 & 3.48 & $5.4-8.0$ & -7.86 & 5.50 & $0.9-1.9$ \\
\hline$C_{1}$ & -7.30 & 2.65 & 6.05 & -7.98 & 3.42 & 6.19 & -7.56 & 5.49 & 1.59 \\
\hline$C_{2}$ & -7.40 & 2.65 & 5.21 & -8.07 & 3.42 & 5.39 & -7.61 & 5.50 & 1.41 \\
\hline$C_{3}$ & -7.29 & 2.65 & 6.06 & -7.98 & 3.42 & 6.22 & -7.54 & 5.49 & $1 . f i 1$ \\
\hline$M_{1}$ & -7.19 & 2.68 & 5.60 & -7.93 & 3.45 & 5.83 & -7.56 & 5.53 & 1.53 \\
\hline$M_{2}$ & -7.34 & 2.67 & 5.90 & -8.03 & 3.44 & 6.08 & -7.61 & 5.52 & 1.58 \\
\hline$W_{1}$ & -8.28 & 2.63 & 5.83 & -8.63 & 3.42 & 5.91 & -7.71 & 5.51 & 1.43 \\
\hline$W_{2}$ & -8.23 & 2.63 & 5.84 & -8.60 & 3.42 & 5.94 & -7.75 & 5.51 & 1.45 \\
\hline$W_{3}$ & -7.98 & 2.67 & 5.23 & -8.47 & 3.44 & 5.45 & -7.72 & 5.55 & 1.33 \\
\hline
\end{tabular}

As can be seen from Table 5 , models $C_{1}$ and $C_{2}$ exhibit a spin-orbit splitting that lies within the band of the experimental uncertainty given in [39]. The single-particle energies of ${ }^{208} \mathrm{~Pb}$ are close to those of the Walcrkis model extended to include non-linear $\sigma^{3}$ and $\sigma^{4}$ terms [4] or the model [5], both for neutrons (Fig. 5) and for protons (Fig. 6). This is encouraging since neither the nucleon/scalar meson nor the nucleon / $\rho$ meson coupling constants can be adjusted to nuclear matter or nuclear properties, in contrast to the Walecka model [4]. Figure 7 shows the binding energy per nucleon, the twonucleon separation energy and the two-nucleon gap for a nucleus with 126 neutrons and different numbers of protons (left) as well as a nucleus with 82 protons and varying numbers of neutrons (right). One perceives that the model fitted to infinite nuclear-matter properties correctly predicts ${ }^{208} \mathrm{~Pb}$ to be a doubly magic nucleus. All these results show that a satisfactory description of finite nuclei is possible within the chiral SU(3) model fitted to nuclear-matter properties. Fits to properties of finite nuclei promise to yield even better rosults [38].

\subsection{Superheavy Nuclei}

Starting from the well-known magic proton and neutron numbers, the question for the next, so far unknown, magic numbers is very important. According to [40] most relativistic mean-field models find the doubly magic nuclei for $Z=120, N=172$ and some predict a doubly magic nucleus with $N=184$ 


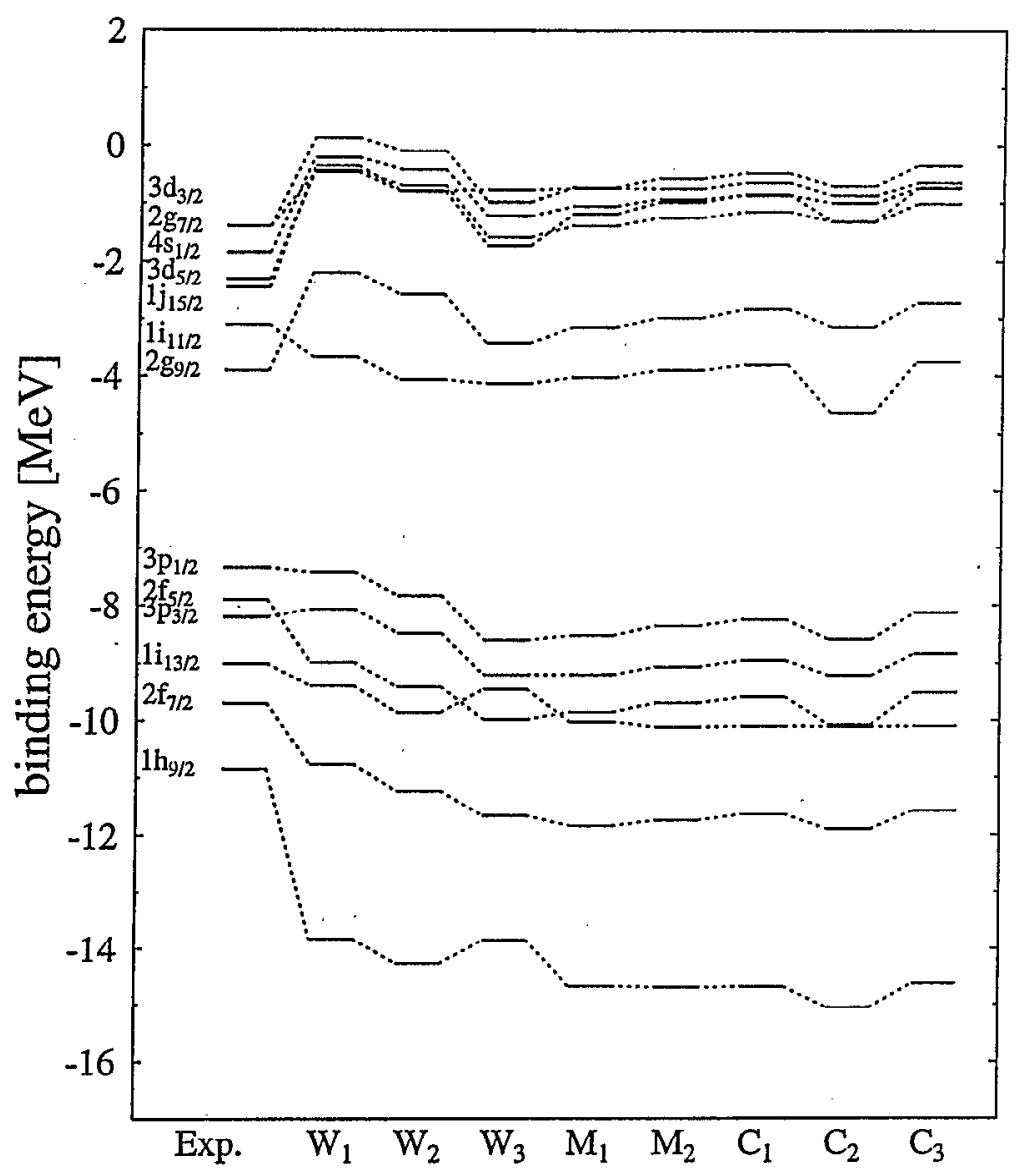

Fig. 5. Single-particle energies of neutrons near the Fermi cnergy in ${ }^{208} \mathrm{~Pb}$. Experimentally measured levels are compared with predictions from various potentials

and $Z=120$. Figure 8 shows the binding energy per particle, the two-nucleon separation energy and the two-nucleon gap around $N=172$ and $Z=120$. The chiral SU(3) model predicts $Z=114$ as a shell closure for a neutron number of 172. One detects a peak at a proton number of 120 as well, but this nucleus is beyond the drip line. Figure 9 shows the same observables like Fig. 8 but for $N=184$ (left) and $Z=120$ (right). One recognizes two possible magic numbers in that region for protons, namely $Z=114$ and $Z=120$. The neutron-shell closures are $N=172, N=184$ and $N=198$. These results should only be seen as preliminary, since on the one hand an improved fitting to doubly magic nuclei and on the other hand the usage of other pair interactions than constant-gap pairing should yield better results.

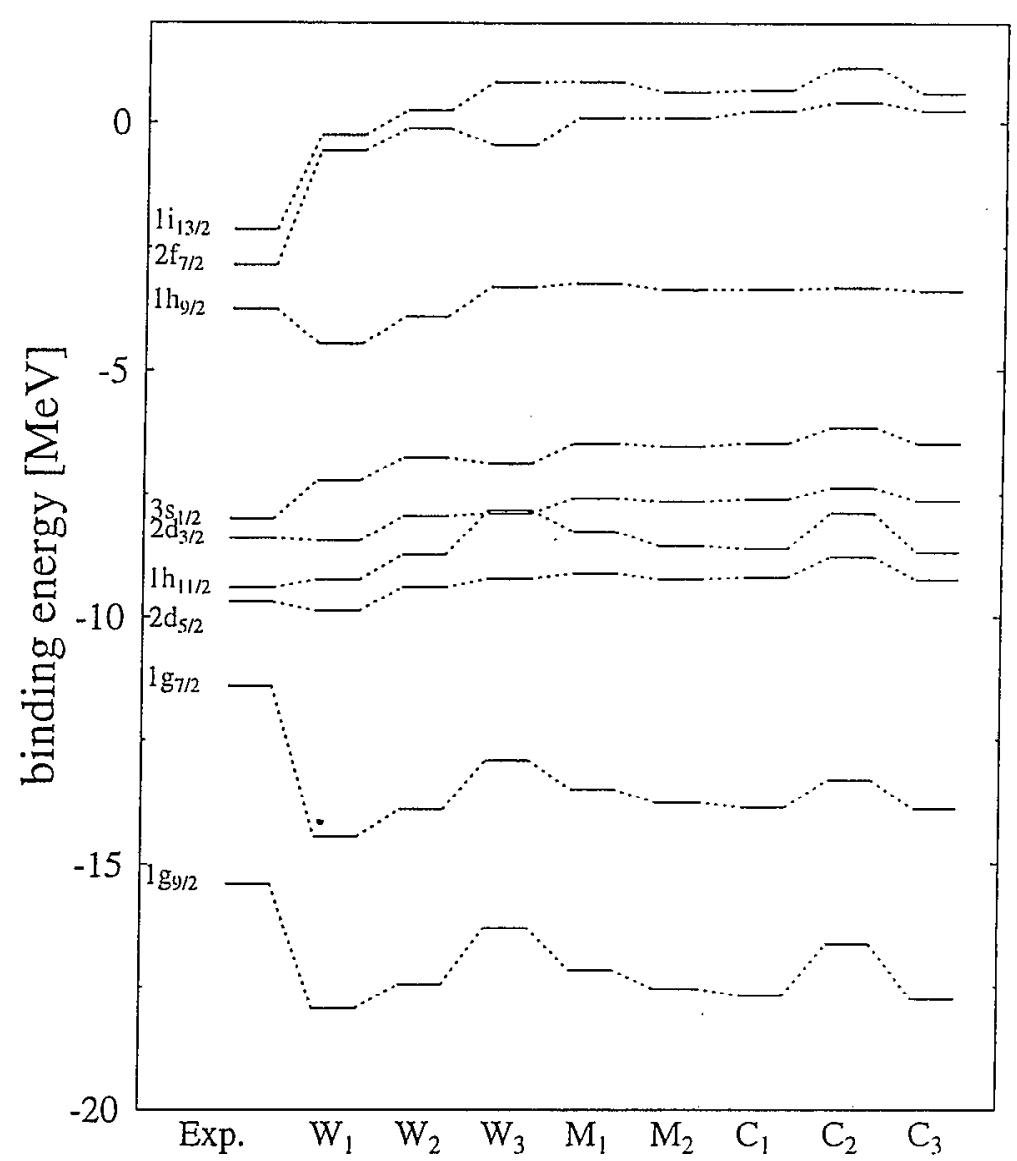

Fig. 6. As for Fig. 5, but for protons

\subsection{Hypernuclei}

We want to compare the experimental hypernuclear diatin with the results obtained using parameter set $C_{1}$. Table 6 shows the experimental $\Lambda$-n-hole one-particle-energy differences for the nuclei ${ }_{A}^{12} \mathrm{C},{ }_{A}^{16} \mathrm{O}$ and ${ }_{A}^{40} \mathrm{Ca}$. from $[41,+2]$. These values are compared to the results from the chiral SU(3) modcl using

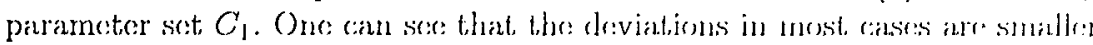
than experimental errors. 'This is even more remarkable, since: the (onulin! constants $g_{\Lambda \sigma}$ and $g_{\Lambda \omega}$ have not been fitted to any hypernuclear datá. But the inclusion of an explicit symmetry-breaking term was necessary to fix the potential depth of a $\Lambda$ particle in infinite nuclear natter to $U_{\Lambda}=-28 \mathrm{Mr} \cdot \mathrm{V}$. Table 6 also shows the results for a Walecka model that was extended to the strange sector [35]. This model yields even better agreement with experiment. but here the coupling constants of the $\Lambda$ were fitted to hypernuclear data. 
$\mathrm{N}=126$
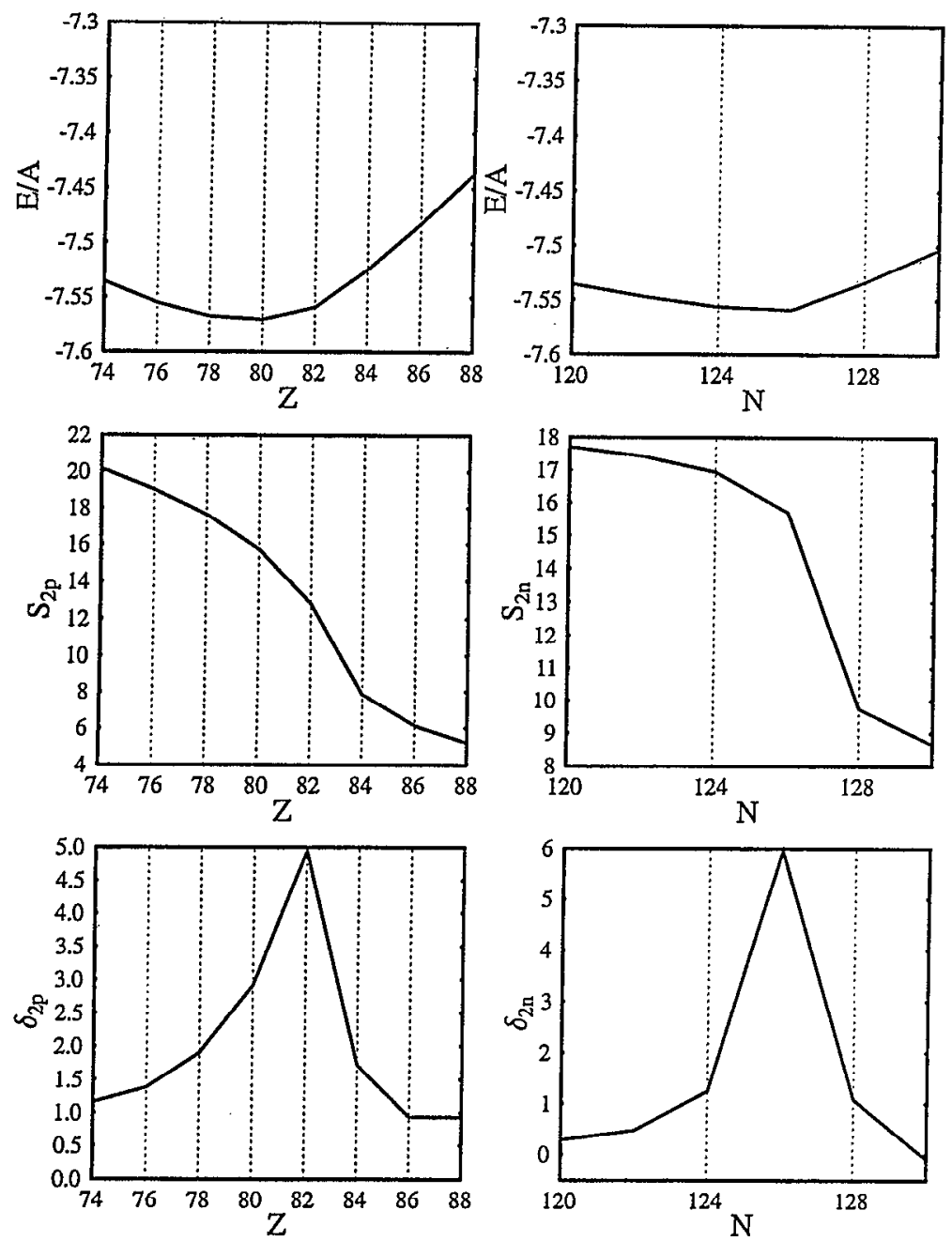

Fig. 7. Shell closures arround ${ }^{208} \mathrm{~Pb}$. (Top) Energy per particle, (middle) twonucleon separation energy and (bottom) two-nucleon gap for different isotopes with (left) $N=126$ and (right) $Z=82$
$\mathrm{N}=172$
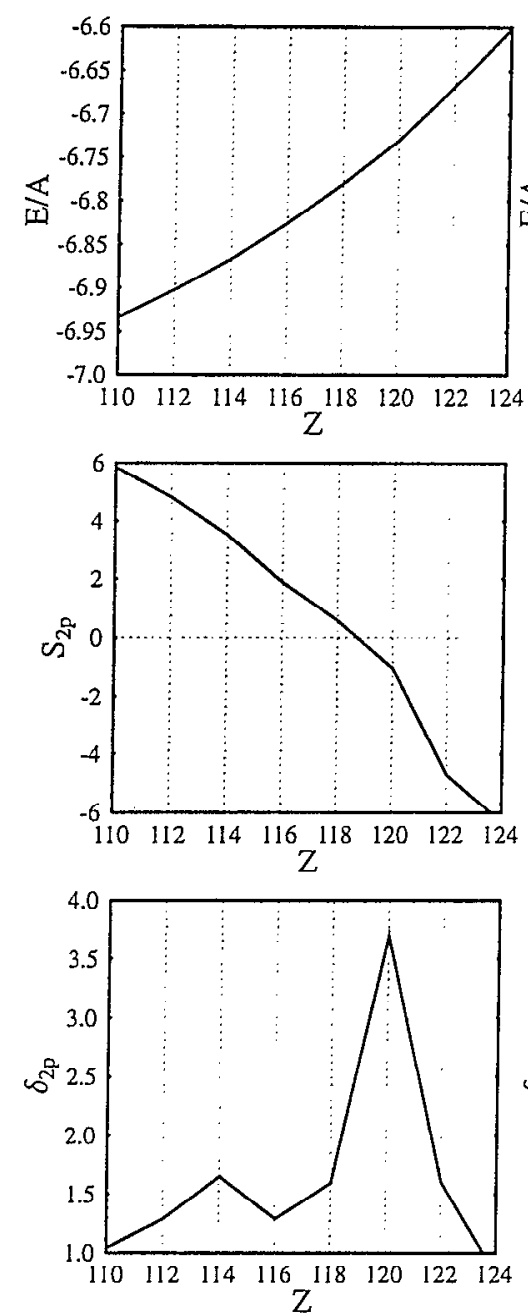

$\mathrm{Z}=114$

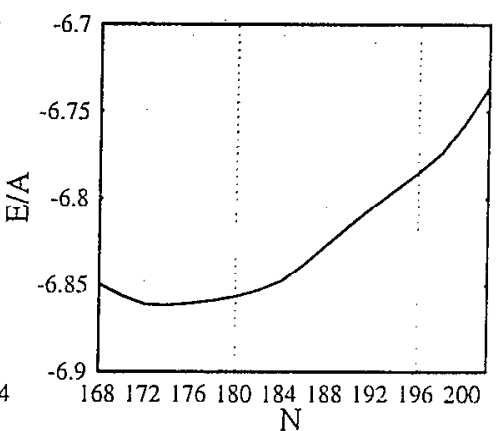

$\mathrm{N}$

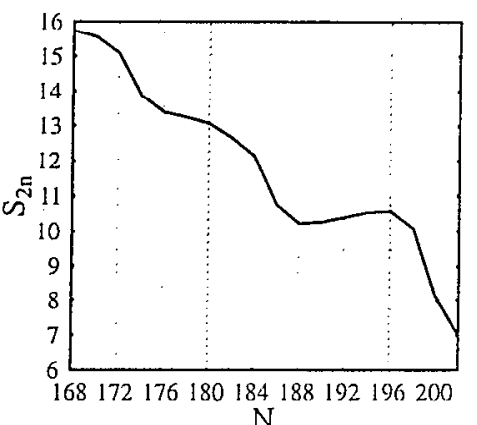

$\mathrm{N}$

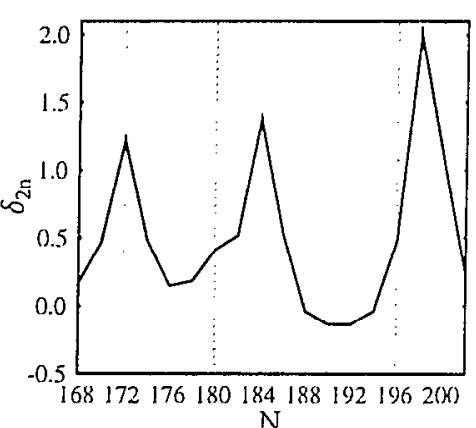

Fig. 8. Superheavy nuclei with $Z=114$ and $N=172$. Same observables as in Irig. $T$ 
$\mathrm{N}=184$
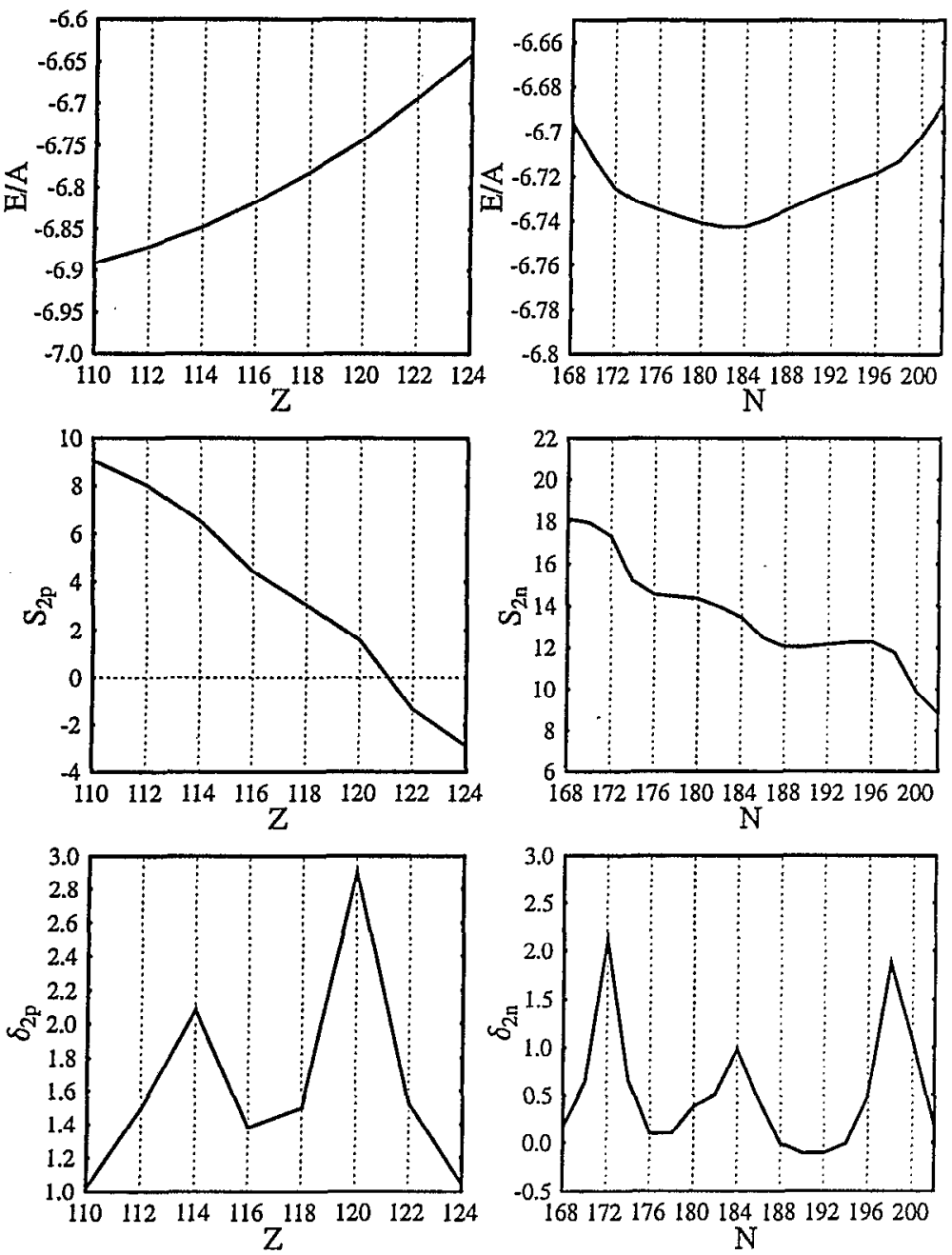

Fig. 9. Superheavy nuclei with $Z=120$ and $N=184$. Same observables as in Fig. 7
Table 6. Experimental $A-n$-hole one-particle-energy-gaps of the nuclei ${ }_{A}^{12} \mathrm{C},{ }_{A}^{10}(\mathrm{O}$ and ${ }_{A}^{40} \mathrm{Ca}$ compared to calculations in the Walecka model [35] and parameter set $C_{1}$ of the chiral SU(3) model. The experimental data was obtained from excitation spectra from $[41,42]$

\begin{tabular}{lccccccc}
\hline & & \multicolumn{3}{c}{ Experiment } & \multicolumn{2}{c}{ Walecka model } & \multicolumn{2}{c}{$C_{1}$ prediction } \\
Kern & Niveaus & $E$ & $\Delta E$ & $E$ & $\Delta E$ & $E$ & $\Delta E$ \\
\hline${ }_{\Lambda}^{12} \mathrm{C}$ & $\left(1 s_{1 / 2}, 1 p_{3 / 2}^{-1}\right)_{\Lambda n}$ & 6.72 & 2 & 5.02 & 1.70 & 7.76 & -1.04 \\
& $\left(1 p_{3 / 2}, 1 p_{3 / 2}^{-1}\right)_{\Lambda n}$ & 18.48 & 2 & 17.21 & 1.27 & 15.78 & 2.7 \\
& & & & & & & \\
${ }_{\Lambda}^{16} \mathrm{O}$ & $\left(1 p_{3 / 2}, 1 p_{3 / 2}^{-1}\right)_{\Lambda n}$ & 19.20 & 2 & 18.88 & 0.32 & 19.29 & -0.09 \\
& $\left(1 p_{1 / 2}, 1 p_{1 / 2}^{-1}\right)_{A n}$ & 13.20 & 2 & 13.89 & -0.69 & 14.36 & -1.16 \\
& $\left(1 s_{1 / 2}, 1 p_{3 / 2}^{-1}\right)_{\Lambda n}$ & 9.90 & 2 & 9.46 & 0.44 & 11.28 & -1.38 \\
& $\left(1 s_{1 / 2}, 1 p_{1 / 2}^{-1}\right)_{\Lambda n}$ & 3.35 & 2 & 3.53 & -0.18 & 5.16 & -1.81 \\
& & & & & & & \\
${ }_{\Lambda}^{40} \mathrm{Ca}$ & $\left(1 p_{1 / 2}, 1 d_{3 / 2}^{-1}\right)_{\Lambda n}$ & 5.79 & 2 & 7.40 & -1.61 & 8.14 & -2.35 \\
& $\left(1 d_{3 / 2}, 1 d_{3 / 2}^{-1}\right)_{A n}$ & 14.47 & 2 & 15.48 & -1.01 & 15.58 & -1.11 \\
& $\left(1 d_{5 / 2}, 1 d_{5 / 2}^{-1}\right)_{\Lambda n}$ & 19.35 & 2 & 20.71 & -1.36 & 20.31 & -0.96 \\
& $\left(1 f_{7 / 2}, 1 d_{5 / 2}^{-1}\right)_{\Lambda n}$ & 28.24 & 2 & 27.14 & 1.10 & 26.49 & 1.75 \\
\hline
\end{tabular}

Figure 10 shows energy levels of $A$ hyperons in various nuclei, as a function of $A^{-2 / 3}$, and these are compared to calculated $A$ one-particle levels for nuclei with $A-1$ nucleons. It can be seen that the results agree well with the experimental data. Furthermore it can be seen that all levels converge to the point $28 \mathrm{MeV}$ for increasing $A$. From this extrapolation the potential clopth of the $A$ in nuclear matter was first deduced [43]. As a further application of the chiral $\mathrm{SU}(3)$ model in the strange sector, Fig. 11 shows the binding energy of baryons in nuclei with different numbers of $\Lambda$ hyperons. It can be seen that with increasing number of $\Lambda$ 's, the hinding mergy first drecreases and then rises again. That is not astonishing, since with adding hyperons a new degree of freedom is introduced. The $\Lambda$ 's are deeper bound than thr lowest-bound nucleons.

\section{Hadronic Matter at High Temperature and Density}

\subsection{Extrapolation to Higher Densities}

Once the parameters have been fixed to nuclear matter at $\rho_{0}$ the condensates and hadron masses at high baryon densities can be investigated. 


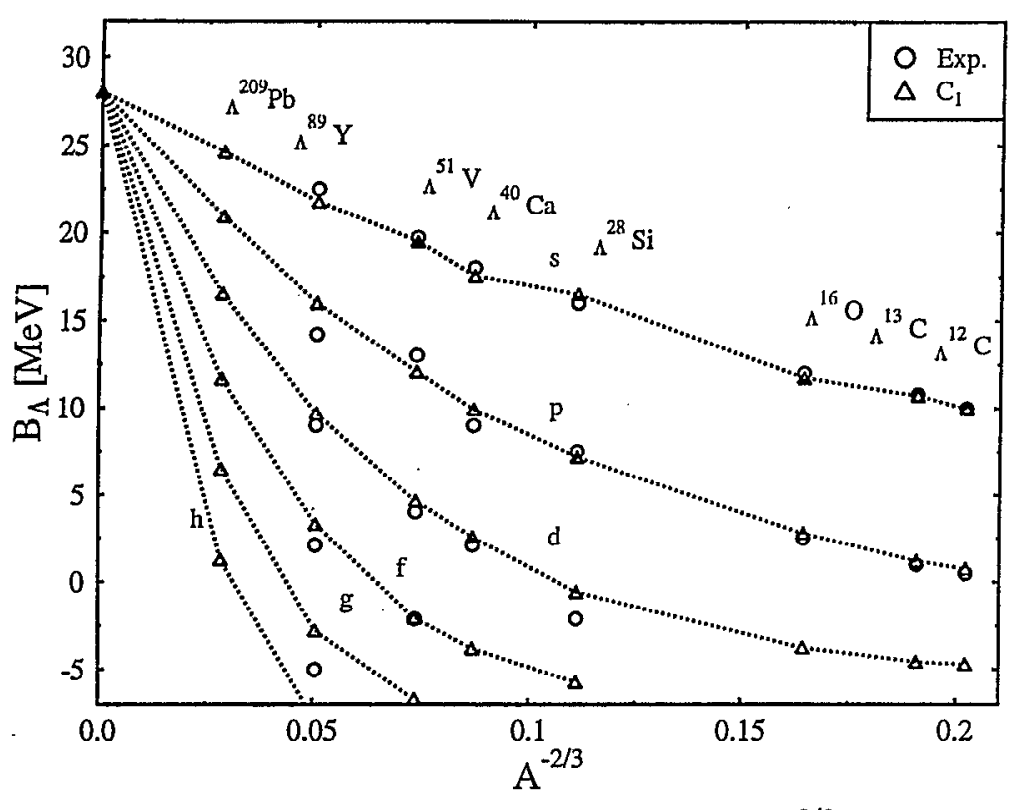

Fig. 10. Energy levels of $\Lambda$ hypernuclei as a function of $A^{-2 / 3}$ compared to experiment [43]. The energy levels converge to the value $-28 \mathrm{MeV}$ for the potential depth of the $\Lambda$ in nuclear matter

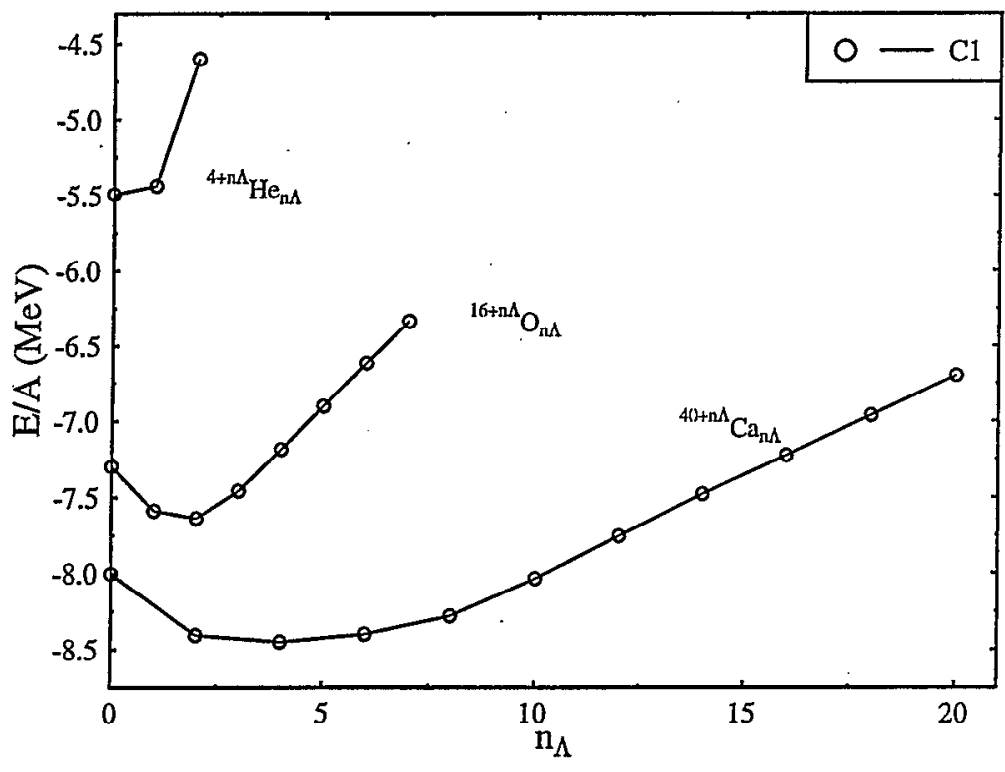

Fig. 11. Binding energy for multi- $\Lambda$ hypernuclei

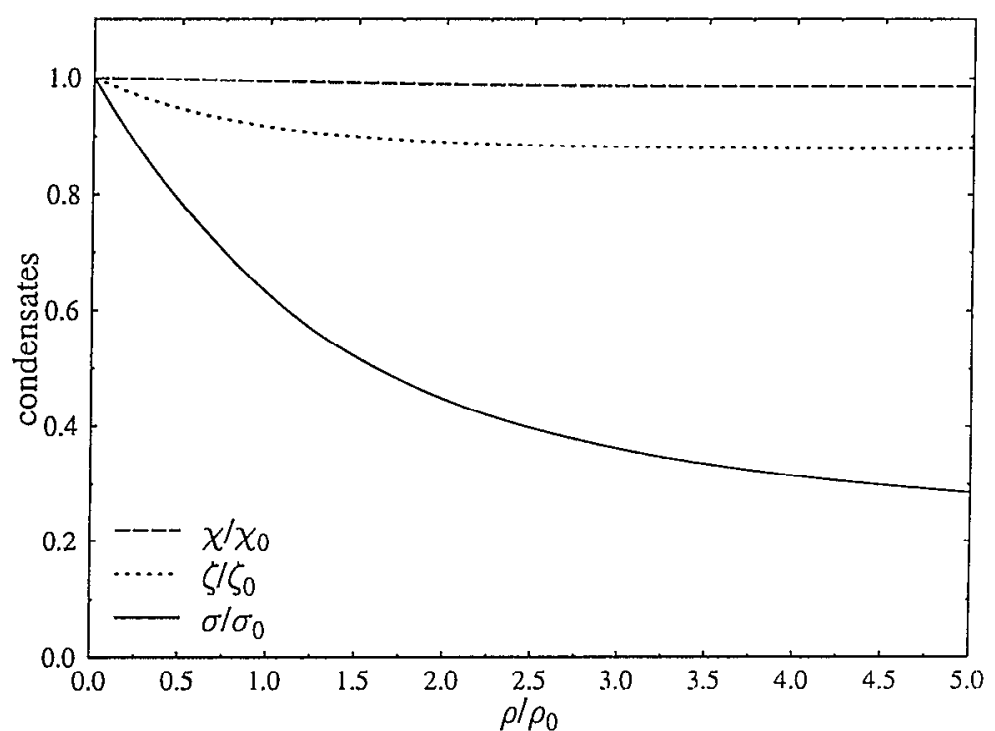

Fig. 12. Scalar condensates $\sigma, \zeta$ and $\chi$ as a function of the baryon density for zero net strangeness

In Fig. 12 we display the scalar mean fields $\sigma, \zeta$ and $\chi$ as a function of the baryon density for vanishing strangeness using parameter set $C_{3}$. Onc sees that the gluon condensate $\chi$ stays nearly constant when the density increases, implying that the approximation of a frozen glueball is reasonable. The strange condensate $\zeta$ is only reduced by about $10 \%$ from its vacuum expectation value. This is not surprising since there are only nucleons in the system and the nucleon- $\zeta$ coupling is fairly weak. The main effect occurs for the non-strange condensate $\sigma$. The field has dropped to $30 \%$ of its vac:unm expectation value at 4 times normal nuclear clensity. If we extrapolate to rven higher densities one observes that the $\sigma$ field does not change significantly: which means all fields saturate around $4 \rho_{0}$. Since the baryon masses are gemerated by the condensates $\sigma$ and $\zeta$, the change of these scalar fields callises the change of the baryon masses in the medium. The density dependencer of the effective spin-1/2 baryon masses is shown for $C_{1}$ in Fig. 13. When the clensity in the system increases, the masses drop significantly up to 4 timc's normal nuclear density. This corresponds to the above-mentioned behavior of the condensates. Furthermore, one observes that the change of the baryon mass depends on the strange-quark content of the baryon. This is caused by the different coupling of the baryons to the non-strange and strange condensates. Figure 14 shows the masses of the vector mesons. They are predicted to stay nearly constant if the density increases. As a further approximation we show in Fig. 15 the nucleon and anti-nucleon potentials as a function of density. Here one can see the very important influence of the quartic vector 


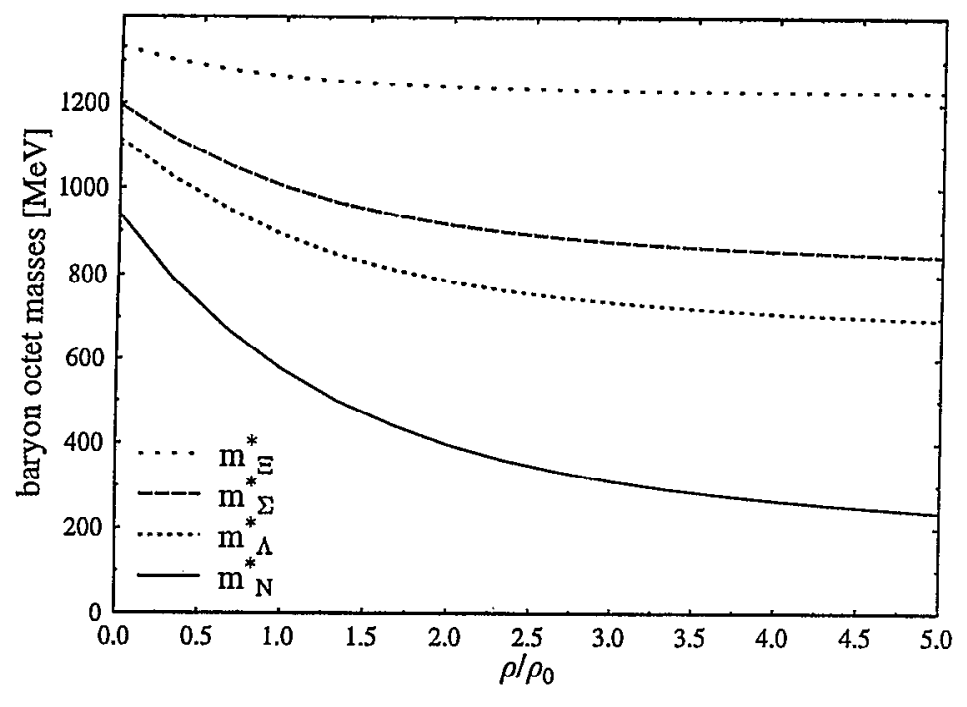

Fig. 13. Effective baryon masses as a function of the baryon density for zero net strangeness

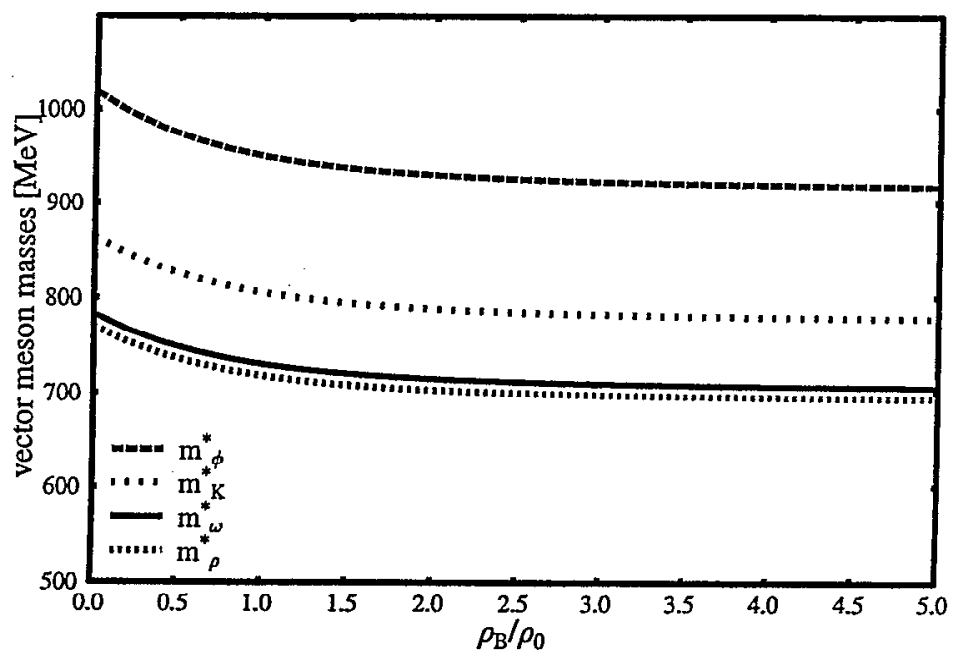

Fig. 14. Vector meson masses as a function of density

meson self-interaction. Including this term, the anti-nucleons become overcritical at densities around $12 \rho_{0}$. If the $\omega^{4}$ term is neglected, anti-nucleons already become critical for $\rho \approx 5 \rho_{0}$. But since this term is essential for the chiral SU(3) model to obtain reasonable values for the effective nucleon mass and the compressibility, this model predicts overcriticality only at very high densities.
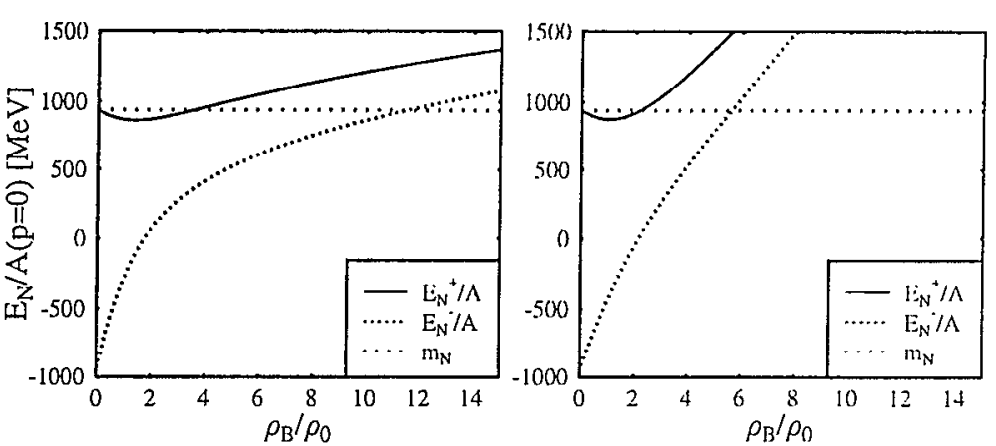

Fig. 15. Nucleon and anti-nucleon energy at $p=0$ as a function of baryon density. On the left-hand side parameter set $C_{1}$ was used, while on the right-hand sidt the coupling constant $g 4$ for the quartic vector meson interaction was set to zero

Now we want to discuss the inclusion of baryonic spin-3/2 resonances and how these affect the behavior of dense hadronic matter. For the following investigations we consider the two parameter sets $C_{1}$ and $C_{2}$, which satisfactorily describe finite nuclei (Sect. 8). As stated before, the main differcnce between the two parameter sets is the coupling of the strange condensate to the nucleon and the $\Delta$. While in $C_{2}$ this coupling is set to zero, in the case of $C_{1}$ the nucleon and the $\Delta$ couple to the $\zeta$ field. This leads to very different predictions for the behavior of dense nuclear matter. In the RMF models both coupling constants of the $\Delta$ baryon are freely adjustable. Ther can be constrained by the fact that there should be, e.g., no $\Delta$ 's in the ground state of normal nuclear matter and a possible second minimum in the nuclear equation of state should lie above the saturation energy of normal nuclear matter. Furthermore QCD sum-rule calculations suggest that the net. attranction for $\Delta$ 's in nuclear matter is larger than that of the nucleon. From these' constraints one can extract a 'window' of possible parameter sets $g_{\Delta \sigma}, g_{\Delta} \mathrm{w}$ [44]. If the masses of the resonances are generated dynamically by the scalar condensate, like in the chiral model, then this coupling constant can be fixed. The vector coupling stays unfixed, but using the constraints from above: om gets only a small region of possible values for $y \Delta \omega$. In Fig. 19 we varied t.lur $\Delta-\omega$ coupling to show the possible range for this value. In all other figures the ratio of the $N-\omega$ coupling to the $\Delta-\omega$ coupling is set to one. In Fig. 16 wor show the equation of state for nuclear matter with and without the $\Delta$ bary"swfor different parameter sets. One sees that at around twice normal nuril $\ldots$ ar density the resonances influence the equation of state. But the strencigth of the influence depends on the strength of the croupling of the nucles, nn tor the strange condensate $\zeta$. This can be understood from Fig. 17 where the ratios of the effective $\Delta$ mass to the effective nucleon mass is displayed. If there is no coupling of the nucleon to the $\zeta$ field $\left(C_{2}\right)$, the mass ratio stays constant butt. if the nucleon couples to the strange condensate $\left(C_{1}\right)$ the situration chander:s. 


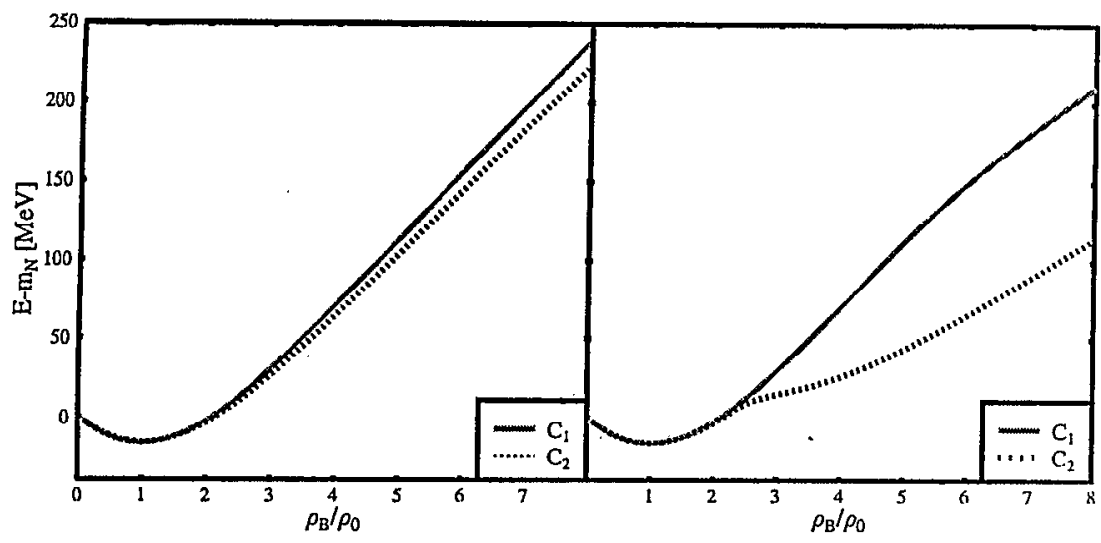

Fig. 16. Equation of state for infinite nuclear matter for the parameter sets $C_{1}$ and $C_{2}$. In the left-hand picture resonances are neglected while they are included in the right-hand picture. If the strange condensate couples to the nucleon and the $\Delta$, the influence of the $\Delta$ resonances on the equation of state is much weaker

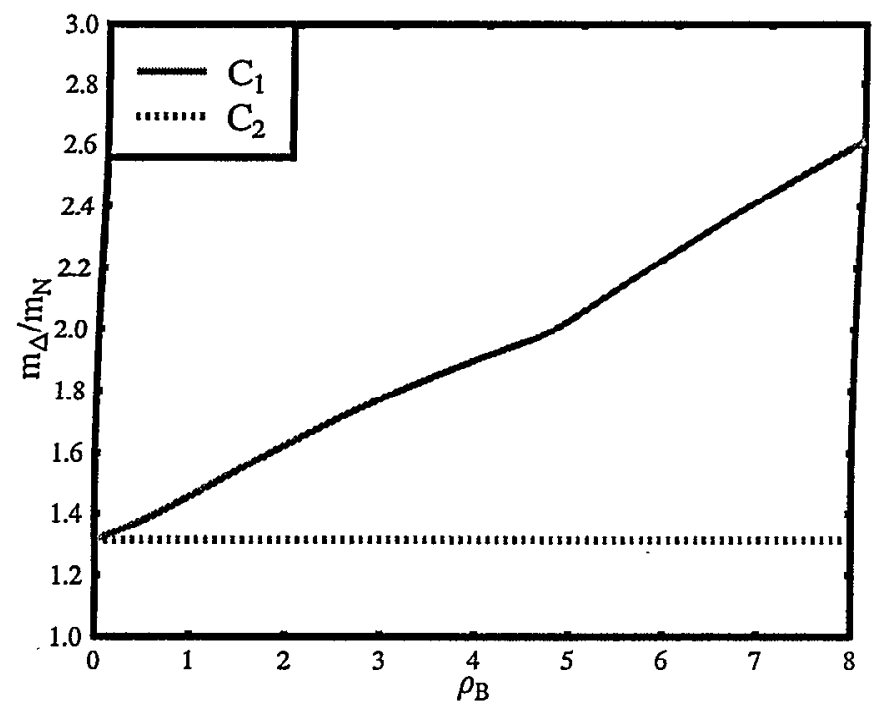

Fig. 17. Ratio of $\Delta$ mass to nucleon mass as a function of density for the two parameter sets $C_{1}$ and $C_{2}$

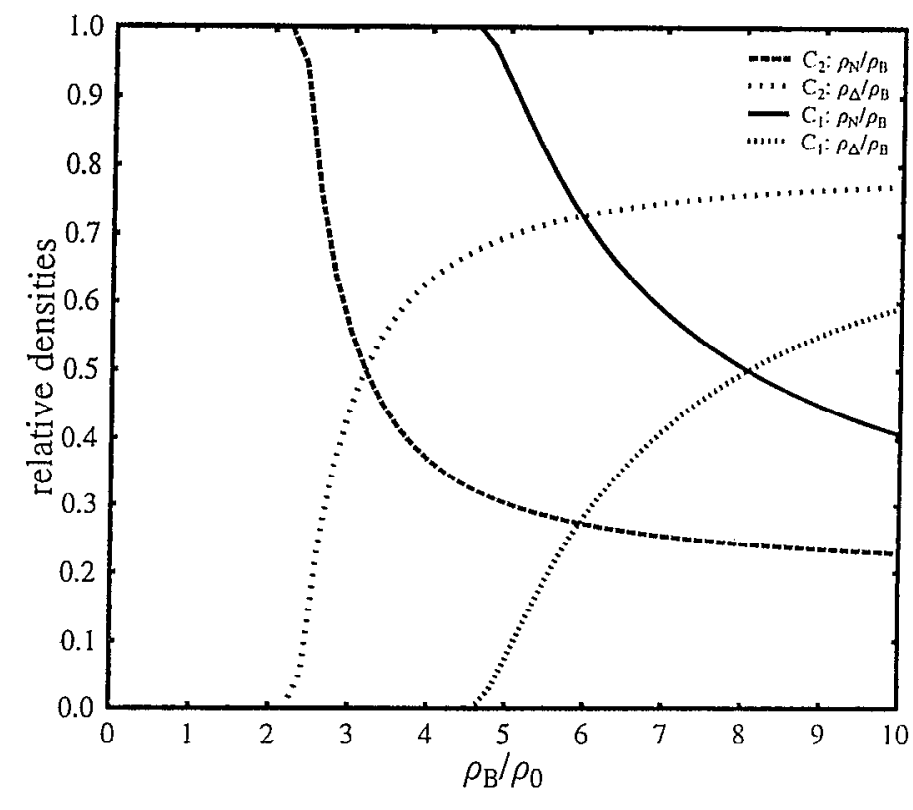

Fig. 18. Relative densities of nucleons and $\Delta$ 's for various parameter sets. The production rate of $\Delta$ 's depends strongly on the parameter set, i.e. on the strength of the nucleon- $\zeta$ and $\Delta-\zeta$ coupling

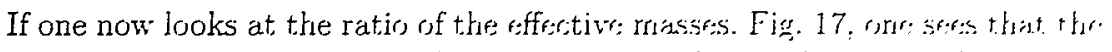
ratio increases with density. That means the nucleon fres:ls mrore sralar attras:tion than the $\Delta$. which leads to reduced $\Delta$ production. as shown in Fig. $1 \times$

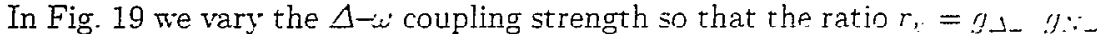
is changed. One observes that a smaller value for this ratio leads re, highr.r $\Delta$ production, since they feel less repulsion, and this leads tos an increasingly softened equation of state. The minimal value for $r_{v}$ is fixed by the corstraint that the second possible minimum in the EOS should not be lower than normal nuclear matter ground state. This implies the minimal value $r_{n}=0.0$. fis $f(n)$ $C_{1}$ and $r_{v}=0.91$ for $C_{2}$. Here one needs further experimental or theoretical constraints to fix this coupling.

\subsection{Extrapolation to Finite Temperatures}

The extrapolation to finite temperatures is straightforward by using the grand canonical ensemble. Now the scalar densities $\rho_{i}^{s}$ and the vector donsitis's $\mu_{1}$ have the form

$$
\rho_{i}^{s}=\gamma_{i} \int_{0}^{\infty} \frac{\mathrm{d}^{3} k}{(2 \pi)^{3}} \frac{m_{i}^{*}}{E_{i}^{*}}\left[\frac{1}{\exp \left[\left(E_{i}^{*}-\mu_{i}^{*}\right) / T\right]+1}+\frac{1}{\exp \left[\left(E_{i}^{*}+\mu_{i}^{*}\right) / T\right]+1}\right],
$$




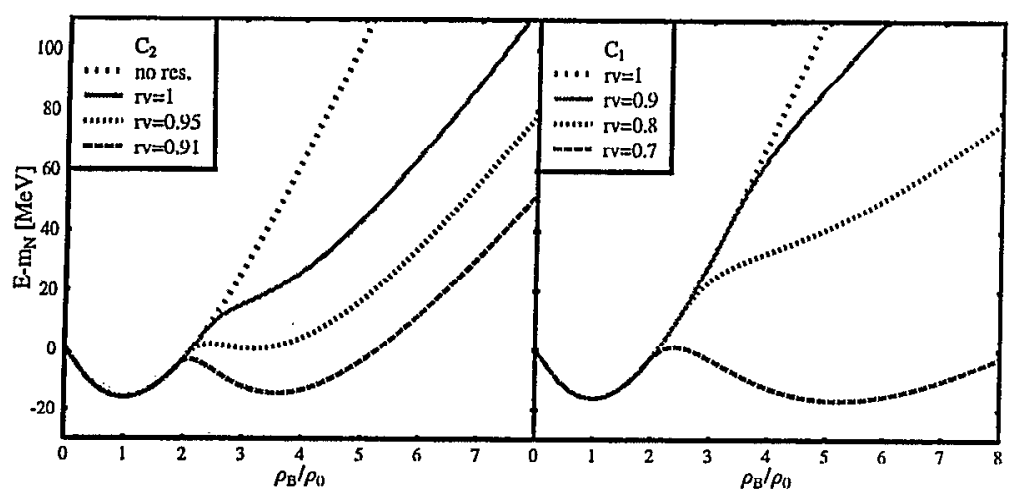

Fig. 19. Equation of state for parameter sets $C_{1}$ and $C_{2}$ for different values of the quotient $r_{v}=g_{N \omega} / g_{\Delta \omega}$. For the $C_{2}$ fit the value of $r_{v}$ should not be less than 0.91 to avoid the density isomer being absolutely stable. For $C_{2}, r_{v}$ must be larger than 0.68

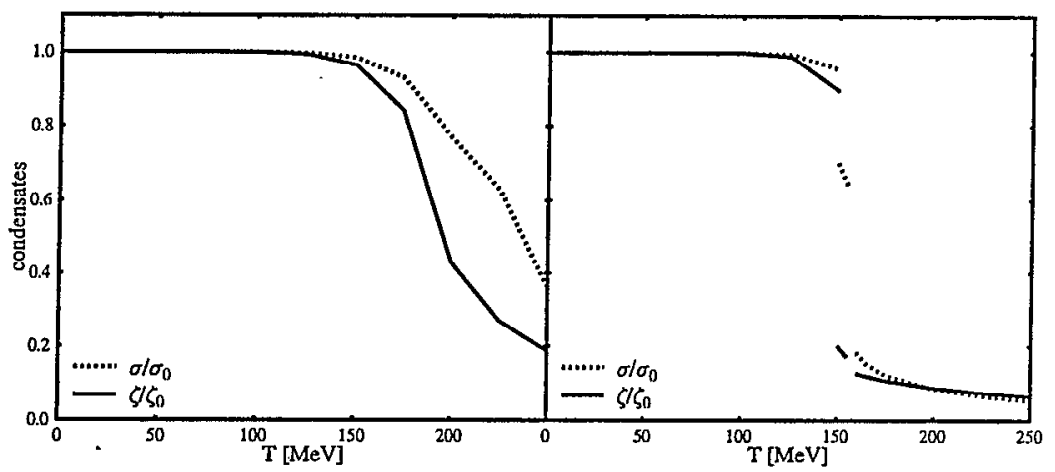

Fig. 20. Chiral condensates as a function of temperature for vanishing chemical potential. (Left) No resonances included. (Right) Baryon decuplet included

$$
\rho_{i}=\gamma_{i} \int_{0}^{\infty} \frac{\mathrm{d}^{3} k}{(2 \pi)^{3}}\left[\frac{1}{\exp \left[\left(E_{i}^{*}-\mu_{i}^{*}\right) / T\right]+1}-\frac{1}{\exp \left[\left(E_{i}^{*}+\mu_{i}^{*}\right) / T\right]+1}\right]
$$

These have to be calculated numerically and inserted in the field equations (42)-(46) to determine the mesonic fields, the grand canonical potential and the thermodynamic quantities for given temperature and chemical potentials.

In Fig. 20 we show the behavior of the strange and non-strange condensates as functions of temperature for vanishing chemical potential with and without baryon resonances. One sees the important influence of the additional degrees of freedom, since through the inclusion of the resonances the ways the chiral condensates change at high $T$ are different. In the case that no resonances are included, one observes a smooth transition to small expectation values of the condensates, while for the case of included resonances

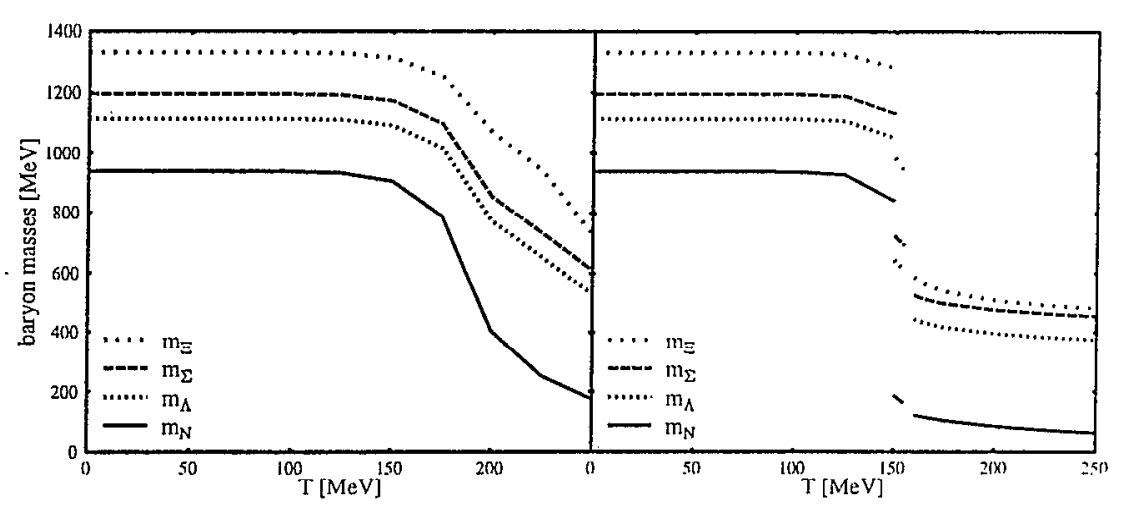

Fig. 21. Baryon octet masses as a function of temperature for vanishing chemical potential. (Left) No resonances included. (Right) Including baryon decuplet

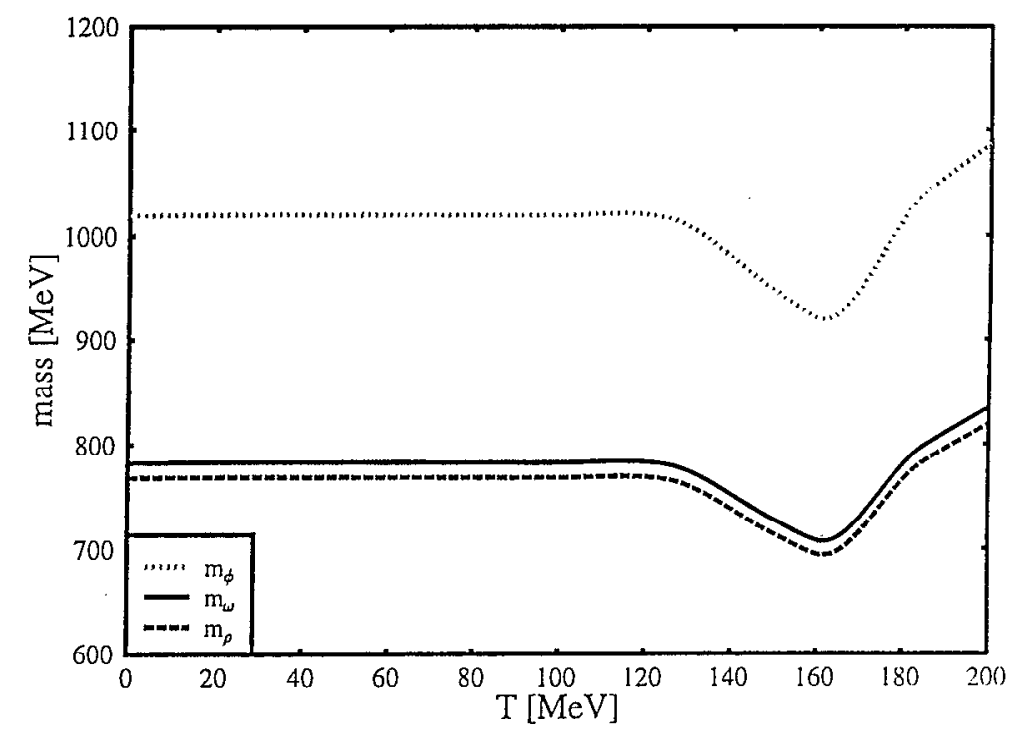

Fig. 22. Vector meson masses as a function of temperature for vanishing chemicul potential

both scalar fields jump to lower values. This is due to the much larger amount. of degrees of freedom which accelerate the process of dropping condensates and increasing scalar density, which leads to further dropping condensiates and again increasing scalar density. This scenario finally leads to a first-order phase transition (actually there are two transitions, one for each scalar ficld. but they are in such a small region, that we will speak only of one transition). The resulting hadron masses as a function of temperature are shown in Figs. 21 and 22 . As a final application we use the obtained temprerature 
and chemical potentials for $\mathrm{S}+\mathrm{Au}$ collisions at an energy of $200 \mathrm{~A} \mathrm{GeV} / c$ as obtained from a thermal model [45] and insert these to obtain the resulting particle ratios. The results in the chiral model, compared to the thermal model and the experimental yields, are shown in Fig. 23. The resulting deviations are enormous. The change of the masses in the hot and dense mcdium (especially the baryon masses) leads to drastically altered particle ratios, and this raises the question of whether at temperatures of more than $150 \mathrm{MeV}$ the thermal model assumptions may be valid.

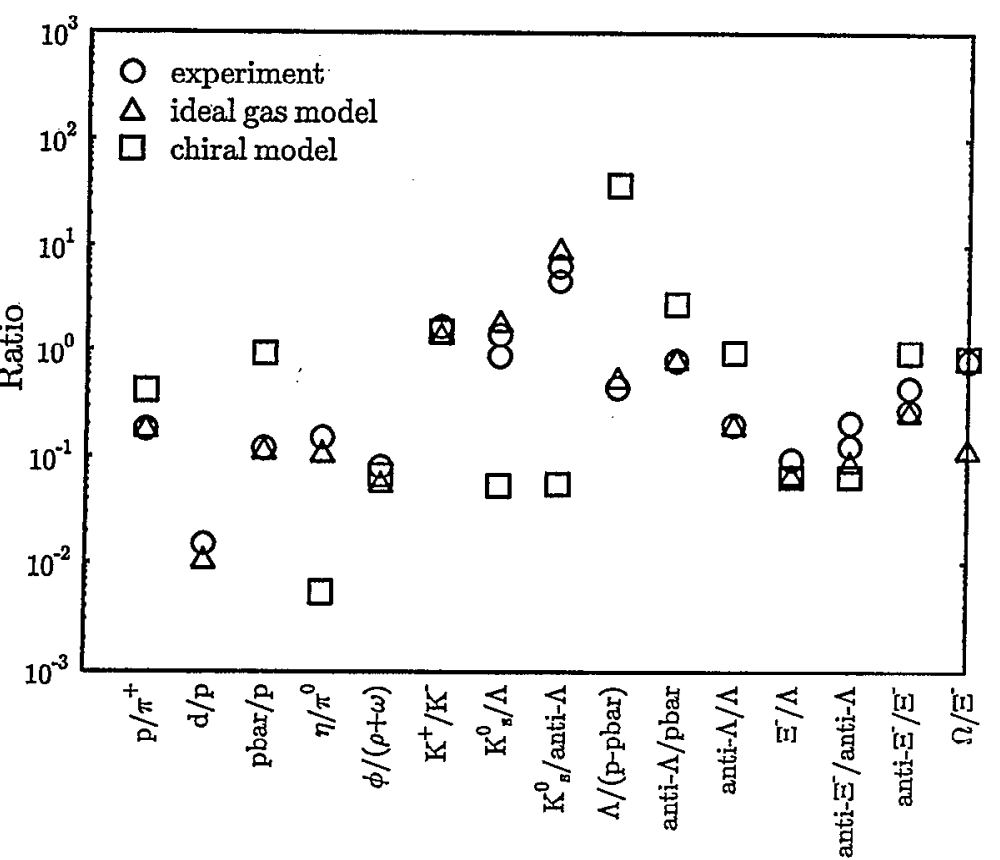

Fig. 23. Particle ratios for $200 \mathrm{~A} \mathrm{GeV} / c \mathrm{~S}+\mathrm{Au}$ collisions using $T=160 \mathrm{MeV}$ and $\mu_{q}=57 \mathrm{MeV}, \mu_{s}=24 \mathrm{MeV}$

\section{Conclusions}

We have reviewed the construction and application of an effective chiral $\mathrm{SU}(3)$ model, which is based on QCD symmetries, namely chiral symmetry and scale invariance. The masses of the hadrons are generated by chiral condensates through the principle of spontaneous symmetry-breaking. Only the pseudoscalar mesons (Goldstone bosons) acquire their mass by explicit symmetry-breaking. Most of the meson-meson and baryon-meson coupling constants are constrained by some hadron masses and basic nuclear-matter saturation properties. As has becn shown, this leads to a model that offers the possibility to reproduce the full range of hadron masses and predict the prop,

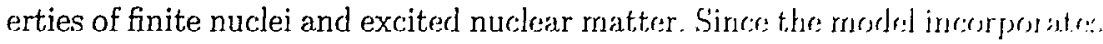
the $\mathrm{SU}(3)$ hadronic multiplets; the extrapolation of the calculations into the strange sector is straightforward for all cases. The quantitative results for finite nuclei are being improved by a direct fit to finite nuclei. Further studir:s are underway to investigate the influence of the predicterl behavirs of hrot and densc nuclear matter on observables in relativistic heavy-ion collisions.

\section{Acknowledgements}

This work was funded in part by the Deutsche Forschungsgemeinschaft (DFG), Gesellschaft für Schwerionenforschung (GSI) and Bundesministerium für Bildung und Forschung (BMBF).

\section{U.S.D.O.E.-DE-ACO2-98CH10886}

\section{Appendix}

The SU(3) matrices of the hadrons are (suppressing the Lorentz indicos)

$$
\begin{aligned}
& X=\frac{1}{\sqrt{2}} \sigma^{a} \lambda_{a}=\left(\begin{array}{ccc}
\left(a_{0}^{0}+\sigma\right) / \sqrt{2} & a_{0}^{+} & \kappa^{+} \\
a_{0}^{-} & \left(-a_{0}^{0}+\sigma\right) \sqrt{2} & \kappa^{0} \\
\kappa^{-} & & \zeta
\end{array}\right), \\
& P=\frac{1}{\sqrt{2}} \pi_{a} \lambda^{a} \\
& =\left(\begin{array}{ccc}
\frac{1}{\sqrt{2}}\left(\pi^{0}+\frac{\eta^{8}}{\sqrt{1+2 w^{2}}}\right) & \pi^{+} & 2 \frac{K^{+}}{w+1} \\
\pi^{-} & \frac{1}{\sqrt{2}}\left(-\pi^{\prime \prime}+\frac{\eta^{\times}}{\sqrt{1+2 w^{2}}}\right) & 2 \frac{K^{\prime \prime}}{w+1} \\
2 \frac{K^{-}}{w+1} & 2 \frac{K^{\prime \prime}}{w+1} & -\frac{\eta^{\mathrm{K}} \sqrt{2}}{\sqrt{1+2 w^{2}}}
\end{array}\right), \\
& V=\frac{1}{\sqrt{2}} v^{a} \lambda_{a}=\left(\begin{array}{ccc}
\left(\rho_{0}^{0}+\omega\right) / \sqrt{2} & \rho_{0}^{+} & K^{*+} \\
\rho_{0}^{-} & \left(-\rho_{0}^{0}+\omega\right) / \sqrt{2} & K^{* 0} \\
K^{*-} & & \frac{K^{* 0}}{\phi}
\end{array}\right) \\
& B=\frac{1}{\sqrt{2}} b^{a} \lambda_{a}=\left(\begin{array}{ccc}
\frac{\Sigma^{0}}{\sqrt{2}}+\frac{\Lambda^{0}}{\sqrt{6}} & \Sigma^{+} & p \\
\Sigma^{-} & -\frac{\Sigma^{\prime \prime}}{\sqrt{2}}+\frac{\Lambda^{0}}{\sqrt{6}} & n \\
\Xi^{-} & \Xi^{0} & -2 \frac{\Lambda^{\prime \prime}}{\sqrt{6}}
\end{array}\right),
\end{aligned}
$$

for the scalar $(X)$, pseudoscalar $(P)$, vector $(V)$, baryon $(B)$ and similarly for the axial vector meson fields. A pseudoscalar chiral singlet $Y=\sqrt{2 / 3} r_{0} \mathbb{1 1}$ (aul 
be added separately, since only an octet is allowed to enter the exponential (13).

The notation follows the convention of the Particle Data Group (PDG) [46], though we are aware of the difficulties of directly identifing the scalar mesons with the physical particles [47]. However, note that there is increasing evidence that supports the existence of a low-mass, broad scalar resonance, the $\sigma(560)$ meson, as well as a light strange scalar meson, the $\kappa(900)$ (see [48] and references therein).

The masses of the various hadrons are generated through their couplings to the scalar condensates, which are produced via spontaneous symmetry breaking in the sector of the scalar fields. Of the nine scalar mesons in the matrix $X$ only the vacuum expectation values of the components proportional to $\lambda_{0}$ and to the hypercharge $Y \sim \lambda_{8}$ are non-vanishing, and the vacuum expectation value $\langle X\rangle$ reduces to

$$
\langle X\rangle=\frac{1}{\sqrt{2}}\left(\sigma^{0} \lambda_{0}+\sigma^{8} \lambda_{8}\right) \equiv \operatorname{diag}\left(\frac{\sigma}{\sqrt{2}}, \frac{\sigma}{\sqrt{2}}, \zeta\right),
$$

in order to preserve parity invariance and assuming, for simplicity, $\mathrm{SU}(2)$ symmetry ${ }^{4}$ of the vacuum.

\section{References}

1. J.D. Walecka, Theoretical Nuclear and Subnuclear Physics, Oxford University Press, New York (1995).

2. B.D. Serot and J.D. Walecka, Adv. Nucl. Phys. 79, 3612 (1997).

3. J. Boguta and A.R. Bodmer, Nucl. Phys. A 292, 413 (1977).

4. R.J. Furnstahl, C.E. Price and G.E. Walker, Phys. Rev. C 36, 2590 (1987).

5. E.K. Heide, S. Rudaz and P.J. Ellis, Nucl. Phys. A 571, 713 (1994).

6. G. Carter, P.J. Ellis and S. Rudaz, Nucl. Phys. A 603, 367 (1996).

7. R.J. Furnstahl, H.B. Tang and B.D. Serot, Phys. Rev. C 52, 1368 (1995).

8. I. Mishustin, J. Bondorf and M. Rho, Nucl. Phys. A 555, 215 (1993).

9. P. Papazoglou, J. Schaffner, S. Schramm, D. Zschiesche, H. Stocker and W. Greiner, Phys. Rev. C 55, 1499 (1997).

10. J. Schechter, Phys. Rev. D 21, 3393 (1980).

11. N.A. Törnqvist, The linear $\mathrm{U}(3) \times \mathrm{U}(3) \sigma$ model, the $\sigma(500)$ and the spontaneous breaking of symmetries, e-print hep-ph/9711483.

12. T. Waas and W. Weise, Nucl. Phys. A 625, 287 (1997).

13. V. Koch, Phys. Lett. B 337, 7 (1994).

14. P. Papazoglou, S. Schramm, J. Schaffner-Bielich, H. Stocker and W. Greiner, Phys. Rev. C 57, 2576 (1998).

15. P. Papazoglou, D. Zschjesche, S. Schramm, J. Schaffiner-Bielich, H. Stocker and W. Greiner, Phys. Rev. C 59, 411 (1999).

4 This implies that isospin-breaking effects will not occur, i.e., all hadrons of the same isospin multiplet will have identical masses. The electromagnetic mass breaking is neglected.
16. R.E. Marshak, N. Mukunda and S. Okubo, Phys. Rep. B 699, 137 (1965).

17. M. Gell-Mann, Physics 1, 63 (1962).

18. B.L. Ioffe, Nucl. Phys. B 188, 317 (1981).

19. G.A. Christos, Phys. Rev. D 35, 330 (1987).

20. C.G. Callan, S. Coleman, J. Wess and B. Zumino, Phys. Rev. 177, 2247 (1969).

21. S. Weinberg, The Quantum Theory of Fields, Vol. 2, Cambridge University Press, Cambridge (1996).

22. V.J.G. Stoks and T.A. Rijken, Nucl. Phys. A 613, 311 (1997).

23. J. Schechter, Y. Ueda and G. Venturi, Phys. Rev. 177, 2311 (1969).

24. J.J. Sakurai, Currents and Mesons, University of Chicago Press, Chicago (1969).

25. S. Gasionowicz and D. Geffen, Rev. Mod. Phys. 41, 531 (1969).

26. P.K. Mitter and L.J. Swank, Nucl. Phys. B 8, 205 (1968).

27. P. Ko and S. Rudaz, Phys. Rev. D 50, 6877 (1994).

28. W.A. Bardeen and B.W. Lee, Phys. Rev. 177, 2389 (1969).

29. R.J. Furnstahl, B.D. Serot and H.B. Tang, Nucl. Phys. A 598, 539 (1996).

30. J. Schechter and Y. Ueda, Phys. Rev. D 3, 168 (1971).

31. B.D. Serot and J.D. Walecka, Int. J. Mod. Phys. E 6, 515 (1997)

32. N.M. Hugenholtz and L. van Hove, Physica 24, 363 (1958).

33. R.J. Furnstahl and B.D. Serot, Phys. Rev. C 47, 2338 (1993).

34. P. Reinhard, Rep. Prog. Phys. 52, 439 (1989).

35. M. Rufa, Ph.D. Thesis, J.W. Goethe Universität, Frankfurt am Main (1989)

36. C.E. Price, J.R. Shepard and J.A. McNeil, Phys. Rev. C 41, 1234 (1990).

37. H. de Vries, C.W. de Jager and C. de Vries, At. Data and Nucl. Data Tables 36,495 (1987).

38. C. Beckmann et al., in preparation (1999).

39. R.J. Furnstahl, J.J. Rusnak and B.D. Serot, Nucl. Phys. A 632, 607 (1998).

40. K. Rutz, Ph.D. Thesis, J.W. Goethe Universität, Frankfurt am Main (1998).

41. W. Brückner et al., Phys. Lett. B 79, 157 (1978).

42. R. Bertini et al., Phys. Lett. B 83, 306 (1979).

43. D.J. Millener, C.B. Dover and A. Gal, Phys. Rev. C 38, 2700 (1988)

44. D. Kosov, C. Fuchs, B. Martemyanov and A. Faessler, Phys. Lett. B 421.37 (1998).

45. P. Braun-Munzinger, J. Stachel, J.P. Wessels and N. Xu, Phys. Lett. B 365. 1 (1996).

46. R.M. Barnett et al., Particle Data Group (PDG), Phys. Rev. D 54, 1 (1996).

47. M. Harada and J. Schechter, Phys. Rev. D 54, 3394 (1996).

48. D. Black, A.H. Fariborz, F. Sannino and J. Schechter, Evidence for a scalar $\kappa(900)$ resonance in $\pi K$ scattering, e-print hep-ph/9804273. 